ARTICLE

https://doi.org/10.1038/s41467-020-14525-4

\title{
FMN reduces Amyloid- $\beta$ toxicity in yeast by regulating redox status and cellular metabolism
}

\author{
Xin Chen (i) ${ }^{1,2}$, Boyang $\mathrm{Ji}^{1,2}$, Xinxin Hao $\mathbb{B}^{3}{ }^{3}$, Xiaowei $\mathrm{Li}^{1}$, Frederik Eisele $\mathbb{D}^{3}{ }^{3}$, Thomas Nyström (i) ${ }^{3} \&$ \\ Dina Petranovic ${ }^{1,2 \star}$
}

\begin{abstract}
Alzheimer's disease (AD) is defined by progressive neurodegeneration, with oligomerization and aggregation of amyloid- $\beta$ peptides ( $A \beta$ ) playing a pivotal role in its pathogenesis. In recent years, the yeast Saccharomyces cerevisiae has been successfully used to clarify the roles of different human proteins involved in neurodegeneration. Here, we report a genome-wide synthetic genetic interaction array to identify toxicity modifiers of $A \beta 42$, using yeast as the model organism. We find that $F M N 1$, the gene encoding riboflavin kinase, and its metabolic product flavin mononucleotide (FMN) reduce $A \beta 42$ toxicity. Classic experimental analyses combined with RNAseq show the effects of FMN supplementation to include reducing misfolded protein load, altering cellular metabolism, increasing $\mathrm{NADH} /\left(\mathrm{NADH}+\mathrm{NAD}^{+}\right)$and $\mathrm{NADPH} /\left(\mathrm{NADPH}+\mathrm{NADP}^{+}\right)$ratios and increasing resistance to oxidative stress. Additionally, FMN supplementation modifies Htt103QP toxicity and $\alpha$-synuclein toxicity in the humanized yeast. Our findings offer insights for reducing cytotoxicity of $A \beta 42$, and potentially other misfolded proteins, via FMN-dependent cellular pathways.
\end{abstract}

\footnotetext{
${ }^{1}$ Division of Systems and Synthetic Biology, Department of Biology and Biological Engineering, Chalmers University of Technology, SE41296 Gothenburg, Sweden. ${ }^{2}$ Novo Nordisk Foundation Center for Biosustainability, Chalmers University of Technology, SE41296 Gothenburg, Sweden.

${ }^{3}$ Institute for Biomedicine, Sahlgrenska Academy, Centre for Ageing and Health-AgeCap, University of Gothenburg, SE40530 Gothenburg, Sweden.

*email: dina.petranovic@chalmers.se
} 
A lzheimer's disease $(\mathrm{AD})$ is the most common neurodegenerative disorder in aging populations. It is categorized as a protein misfolding, or protein conformational disease due to the accumulation of misfolded amyloid- $\beta(A \beta)$ peptides, which are defined as one of its principal hallmarks ${ }^{1}$. The aggregation of $\mathrm{A} \beta$ is thought to be an early driver of $\mathrm{AD}$, triggering a cascade of pathogenic processes, such as inflammation, neurofibrillary tau-tangle formation, and synapse dysfunction ${ }^{2}$. Brain extracts from $\mathrm{AD}$ patients or transgenic mice containing $\mathrm{A} \beta$ have also shown to accelerate cerebral $A \beta$ plaque accumulation and associated pathology in genetically modified mice ${ }^{3}$. A $\beta 42$, which is two amino acids longer than the usual form of the peptide $(\mathrm{A} \beta 40)$, is more hydrophobic and prone to form aggregates, being enriched in patients diagnosed with $\mathrm{AD}^{4}$. Nonetheless, despite our understanding of $\mathrm{AD}$ pathogenesis tremendously advancing over the past three decades, efforts to translate this knowledge into effective therapies have, so far, had limited success ${ }^{5}$.

As a principal organelle responsible for protein quality control and secretion, the endoplasmic reticulum (ER) is dramatically perturbed in $\mathrm{AD}$ neurons ${ }^{6}$. Increased $\mathrm{A} \beta$ production and/or aggregation is proposed to result in abnormal levels of ER stress, contributing towards synapse dysfunction in $\mathrm{AD}^{7}$. To restore cellular proteome homeostasis (proteostasis), ER stress can activate the unfolded protein response (UPR) ${ }^{8}$, which in turn promotes the proper folding of misfolded proteins and suppresses translation, preventing an overload of the ER with newly synthesized proteins. As well, UPR leads to an activation of protein degradation via the ER-associated protein degradation (ERAD) pathway ${ }^{9,10}$. However, under chronic or irreversible ER stress conditions, the UPR shifts its signaling toward cell death ${ }^{11}$. Modulation of ER-UPR has therefore been one potential therapeutic strategy for slowing down $\mathrm{AD}$, using gene therapy or pharmacological approaches ${ }^{12,13}$.

Due to the strong conservation of protein quality control systems among eukaryotic cells, the yeast Saccharomyces cerevisiae has become a powerful model organism to study misfolded proteins and their implication in human pathologies. Many available molecular and genetic tools, alongside defined media, and ease of handling provide many benefits for using this model system for high-throughput genetic and drug candidate screenings ${ }^{14,15}$. Indeed, several humanized yeast models have been successfully developed to study different aspects of $A \beta$ toxicity. In humans, $A \beta$ peptides are generated through the sequential cleavage of the amyloid precursor protein (APP) ${ }^{16}$ by BACE and $\gamma$-secretase ${ }^{17}$, which occurs in the secretory pathway and endocytic pathway in neurons ${ }^{18}$. In yeast, these human $A \beta$ peptides can be co-expressed with an ER Kar2 signal peptide, to ensure that $A \beta$ peptides are transited through the secretory pathway and exported from the cytoplasm. However, as the yeast cell wall prevents secreted $A \beta$ from diffusing away extracellularly, these $A \beta$ peptides re-enter into the cell through endocytosis. A 342 peptides subsequently form more oligomers relative to $\mathrm{A} \beta 40$ and exhibit an increased cellular toxicity in yeast ${ }^{19,20}$.

To mimic chronic cytotoxicity during $\mathrm{AD}$ progression, we recently developed constitutive $\mathrm{A} \beta 42$ and $\mathrm{A} \beta 40$ expression models in yeast ${ }^{21}$. The constitutive $A \beta 42$ expression provides a model for moderate and cumulative effects of $A \beta 42$ toxicity, over a longer period, in conditions of aging yeast cells. Similar to previous observations in human neurons and other $\mathrm{AD}$ model organisms $^{22}$, we found that constitutive expression of $A \beta 42$ peptides forms more toxic oligomers and triggers a stronger ER stress response and UPR, compared to the expression of less toxic A $\beta 40$ peptides ${ }^{23}$. We also observed reduced mitochondrial function and an increase in the production of reactive oxygen species (ROS), alongside a redirection of energy from cell growth to maintenance ${ }^{23}$.
The synthetic genetic array (SGA) approach has been applied to yeast neurodegeneration models, such as AD, Huntington's disease (HD) ${ }^{24}$ and Parkinson's disease (PD) ${ }^{25}$, demonstrating its robust capability to help identify genes and mechanisms underlying phenotypes of interest, such as modeling toxicity of human disease proteins. In previous studies, the nonessential genomewide deletion library has been applied to $A \beta 42$ expression strain to identify proteins and cellular processes affecting intracellular $A \beta 42$ aggregation and toxicity ${ }^{26,27}$. The screening of GFP fused A $\beta 42$ (A $\beta 42-G F P$ ) identified four major cellular processes as being significantly overrepresented: mitochondrial function, phospholipid metabolism, transcriptional/translational regulation and inositol biosynthesis ${ }^{26}$. Another screening of A $\beta 42$ strain with a $\alpha$-prepro factor sequence revealed that ER-Golgi traffic, mitochondrial functioning, cell cycle, DNA replication stress response and ESCRT machinery play vital roles in A $\beta 42$ toxicity $^{27}$. In both studies, expression of $A \beta 42$ peptides is under the control of a strong inducible promoter, whereas the A $\beta 42$ production in neurons is constitutive. The inducible promoter allows for well-timed induction of acute cytotoxicity but is accompanied by a drastic change in carbon-source and metabolism.

Here we take advantage of our improved $A \beta 42$ humanized yeast model to investigate genetic interactions that can provide insights into the A $\beta 42$ cytotoxic phenotype. We applied SGA technology to screen for genetic mutants with an altered A $\beta 42$ sensitivity profile, by using a yeast deletion mutant library in which we constitutively expressed $A \beta 42$. The two mutant libraries comprise a collection of $\sim 4300$ deletion strains for nonessential genes (SGA-V2) and $\sim 1200$ temperature sensitive mutant strains for essential genes (ts-V6). These two sets together represent more than $80 \%$ of all yeast genes ${ }^{28}$. Compared with previous studies, inclusion of essential genes screening in our SGA analysis may further exploit the cellular mechanisms affecting A $\beta 42$ toxicity. This unbiased yeast genome-scale screen thus enables a global analysis of synthetic genetic interactions, which enabled us to identify mechanistic connections between genes and their corresponding pathways ${ }^{29}$. Using the tool in this study, it was therefore possible to map the genome-wide interaction networks involved in A $\beta 42$ toxicity. From the essential mutant library, we identified the riboflavin kinase gene (FMN1) and its metabolic product flavin mononucleotide (FMN) as being able to alleviate cellular $\mathrm{A} \beta 42$ toxicity.

\section{Results}

Yeast genome-wide screening for modulators of $\mathrm{A} \beta 42$ toxicity. Our humanized $A \beta 42$ yeast model was constructed with a centromeric plasmid under the control of a constitutive GPD promoter, with the Kar2 signal sequence in front of the A $\beta 42$ sequence ${ }^{21}$. To minimize the experimental variation during high-throughput screening, we integrated two copies of the Kar2A $\beta 42$-encoding sequence under the control of the GPD promoter into the SGA starting strain (Y7092) (Supplementary Fig. 1), and tested it to confirm that the previously characterized phenotypes were preserved. The main reason for using the Y7092 strain being that it incorporates reporters and markers necessary for SGA haploid strain selection, following meiotic recombination. To create an SGA control query strain, only the promoter and terminator sequences were integrated into the Y7092 strain (Supplementary Fig. 1). The query strains were mated with the yeast deletion mutant library to generate two arrays, where each deletion strain on the array was combined with either the control or A $\beta 42$ expression. The scores for growth defects were generated through comparing the colony sizes of mutants with the $A \beta 42$ expression to mutants with the control (Fig. 1a). Scores $<0$ or $>0$ 

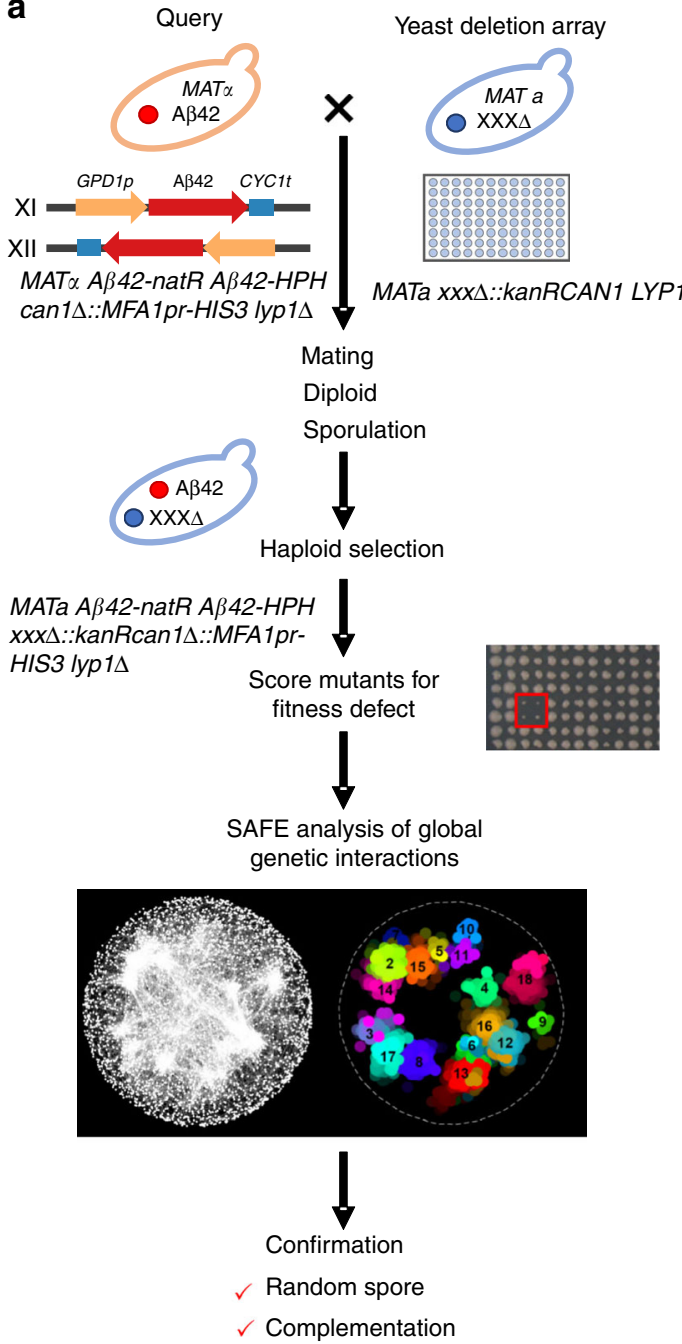

b

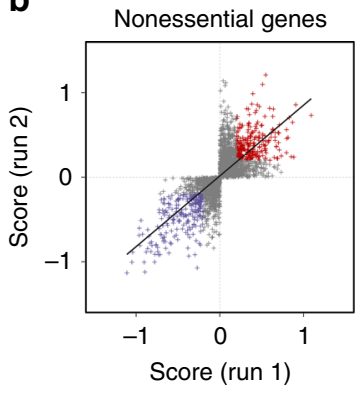

C

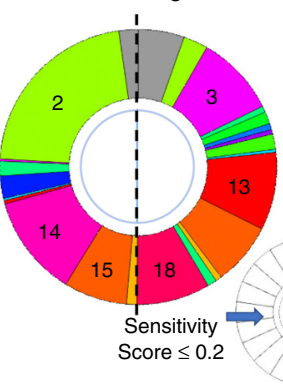

\begin{tabular}{llllllll|l|lllllllllll}
1 & 2 & 3 & 4 & 5 & 6 & 7 & 8 & 9 & 10 & 11 & 12 & 13 & 14 & 15 & 16 & 17 & 18
\end{tabular}

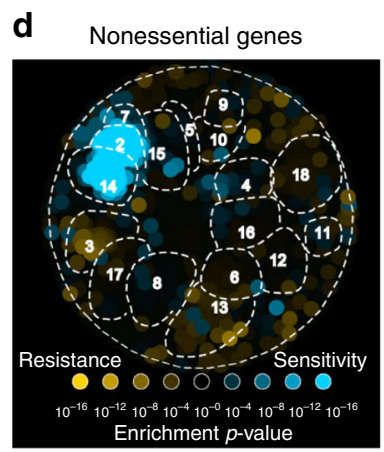

Essential genes

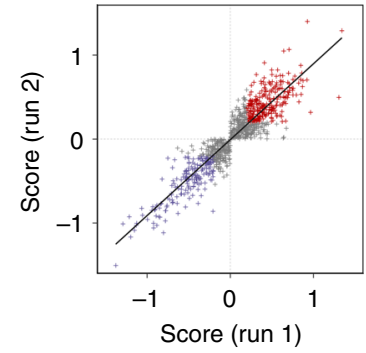

Essential genes

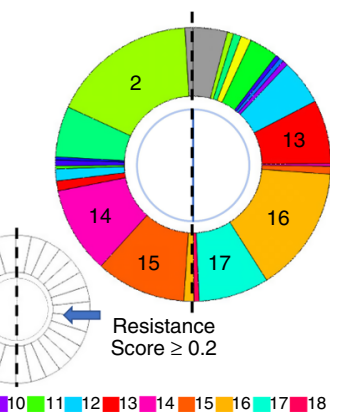

Essential genes

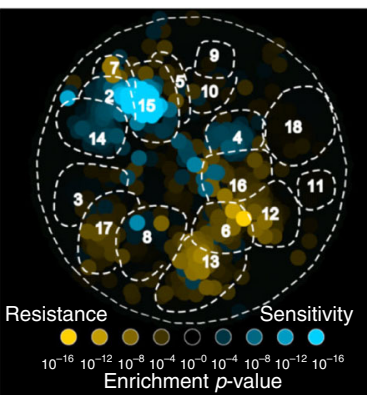

Fig. 1 Genome-wide synthetic genetic array (SGA) screen to identify modulators of A $\mathbf{\beta 4 2}$ toxicity. a $A$ schematic workflow for identifying mutants that can alter $A \beta 42$ toxicity. $\mathbf{b}$ Scores for each genetic mutant, from two independent experiments, with those that significantly change $A \beta 42$ toxicity indicated in red ( $p$-adj $<0.05$, score $\geq 0.2$ ), and blue ( $p$-adj $<0.05$, score $\leq-0.2$ ), respectively. c Gene ontology (GO) biological process term distributions among mutant strains with significantly affected $A \beta 42$ toxicity ( $p$-adj $<0.05$, score $\geq 0.2$ or $\leq-0.2$ ). Each number corresponds to a different biological process $G O$ term as follows: 1. Other; 2. Glycosylation, protein folding/targeting, cell wall biosynthesis; 3. Ribosome biogenesis; 4. Protein degradation; 5. Cytokinesis; 6. Nuclear-cytoplasmic transport; 7. Multivesicular body (MVB) sorting and pH-dependent signaling; 8. mRNA processing; 9. tRNA wobble modification; 10. Peroxisome; 11. Metabolism and fatty acid biosynthesis; 12 . DNA replication and repair; 13 . Transcription and chromatin organization; 14 . Vesicle traffic; 15. Cell polarity and morphogenesis; 16 . Mitosis and chromosome segregation; 17. rRNA and ncRNA processing; 18. Respiration, oxidative phosphorylation, mitochondrial targeting. The top $3 \mathrm{GO}$ terms are indicated as number in each individual category. $\mathbf{d}$ Spatial analysis of functional enrichment of mutants with significantly ( $p$-adj $<0.05$ ) affected $A \beta 42$ toxicity and score $\geq 0.2$ or $\leq-0.2$. Blue colored points indicate mutants more sensitive to $A \beta 42$ toxicity (score $\leq-0.2$ ) and yellow color indicates mutants that are more resistant (score $\geq 0.2$ ), as measured via their growth during SGA screening. GO biological process terms are indicated as in $\mathbf{c}$ and outlined by dashed lines.

represent either a decrease or an increase of growth in mutants with A $\beta 42$ expression.

The SGA screen was performed independently twice. The cutoffs for scores were set at $\leq-0.2$ or $\geq 0.2$ ( $p$-adj $<0.05$, Supplementary Fig. 2 and Supplementary Fig. 3). Among the analyzed 4,300 mutants for nonessential genes, 229 were scored $\leq-0.2$, that is these mutants showed higher sensitivity to $\mathrm{A} \beta 42$ toxicity and 259 were scored $\geq 0.2$, that is these mutants showed higher resistance to A $\beta 42$ toxicity, in both SGA tests as seen through their difference in growth profiles (Fig. $1 \mathrm{~b}$ and Supplementary Data 1). The analysis of the 1,200 mutants for essential genes revealed 198 to have a score $\leq-0.2$ and 253 had a score $\geq 0.2$ (Fig. $1 \mathrm{~b}$ and Supplementary Data 2). The gene set enrichment analysis (GSEA) showed that sensitive mutants (decreased growth with $\mathrm{A} \beta 42$ expression, score $\leq-0.2)$ significantly enriched in the vesicle-mediated transport $(p=7.94 \mathrm{e}-10)$, glycosylation $(p=8.01 \mathrm{e}-5)$ and response to unfolded protein $(p=9.63 \mathrm{e}-6)$ in nonessential collection (Supplementary Fig. 4). While in essential collection, the sensitive mutants significantly enriched in the vesicle-mediated transport $(p=1.55 \mathrm{e}-8)$, protein $\mathrm{N}$-linked glycosylation $(p=4.58 \mathrm{e}-6)$ and autophagy $(p=2.52 \mathrm{e}-4)$ (Supplementary Fig. 5). Furthermore, we performed a functional enrichment analysis via spatial analysis of functional enrichment $(\mathrm{SAFE})^{30}$. The enrichment of GO terms suggested that $44 \%$ and $34 \%$ of the genes mutated in the sensitive mutants in nonessential and essential collections, respectively, belonged to group 2 (glycosylation, protein folding/targeting, cell wall biosynthesis processes) (enrichment $p$ value $<0.05$ ). Similarly, $24 \%$ and $21 \%$ of 
the genes mutated in the sensitive mutants in nonessential and essential collections, respectively, were represented by group 14 (vesicle traffic processes) (enrichment $p$ value $<0.05$, Fig. 1c, d). Moreover, the enrichment of GO terms for resistant mutants that had increased growth with $\mathrm{A} \beta 42$ expression (score $\geq 0.2$ ) was represented in different biological processes in both mutant collections (Fig. 1c, d). Besides these results, among the essential genes required for $\mathrm{A} \beta 42$ tolerance, many belonged to group 4 (protein degradation processes) as well as group 15 (cell polarity and morphogenesis processes). Essential genes, which mutations caused increased resistance against $A \beta 42$, were enriched in group 13 (transcription and chromatin organization processes), group 16 (mitosis and chromosome segregation processes) and group 17 (rRNA and ncRNA processing processes) (enrichment $p$ value $<$ 0.05 , Fig. 1c, d). The complete list of GO terms from SAFE analysis can be found in Supplementary Tables 1 and 2 .

Yeast mutants with increased sensitivity to $A \boldsymbol{\beta} 42$ toxicity. Gene set enrichment analysis revealed that $A \beta 42$ toxicity was found to be increased in mutants involved in protein secretion and degradation processes, including protein translocation, posttranslational modifications, folding to a mature protein, ER to Golgi transport, ER to vacuole transport, ERAD and autophagy. To visualize the functions of these mutated genes, we mapped all mutants that met our significance and growth score cut offs $(p$-adj $<0.05$, score $\leq-0.2$ or $\geq 0.2)$ on a schematic representation of protein secretion and degradation pathways map (Fig. 2a). These findings are in accordance with our previous findings from genome-wide transcriptional analysis, which showed that $\mathrm{A} \beta 42$ expression triggers a strong ER stress, resulting in activation of the UPR to upregulate processes involved in protein folding/ maturation, ER-to-Golgi trafficking and ERAD ${ }^{23}$ (Supplementary Fig. 6).

By selecting more stringent cutoff values ( $p$-adj $<0.05$, score $\leq$ -0.5 or $\geq 0.5)$, 47 sensitive mutants and 7 resistant mutants were identified (listed in Supplementary Tables 3 and 4, respectively). Interestingly, 35 out of these 54 mutants were determined in human (HomoloGene, https://www.ncbi.nlm.nih.gov/homologene). To assess the potential clinical relevance of these genes, we further examined the mRNA expression of the corresponding human orthologs in the prefrontal cortex of $\mathrm{AD}$ patients versus controls (healthy) samples, which enabled us to examine 28/35 human ortholog genes. We discovered that 14 and 8 out of the 28 human orthologs showed significantly decreased and increased mRNA levels, respectively (Fig. 2b and Supplementary Fig. 7). We further manually tested the phenotypes of the 28 corresponding yeast mutants, of which 13 mutant phenotypes were confirmed by random spore test (Table 1).

Six out of these 13 mutants had functions related to ER and glycosylphosphatidylinositol (GPI) biosynthesis (FMN1, YIP1, CNE1, ERG27, GPI13, and GPI1), four were related to glycosylation and translocation (WBP1, OST1, OST3, SEC63), and three were functionally associated with COPI transport (SEC21, ERD2, RER1). Interestingly, of these human orthologs, four (SEC6331, $K D E L R 1^{32}, R E R 1^{33}$, and $\left.R P N 1^{34}\right)$, which are the orthologs of yeast SEC63, ERD2, RER1, and OST1, respectively, have already been identified in previous studies for association with $\mathrm{AD}^{31-34}$.

Defects in riboflavin metabolism increases $\mathrm{A} \beta 42$ toxicity. Among these confirmed mutants with human orthologs, we found the FMN1 gene. FMN1 encodes an essential enzyme that is responsible for catalyzing the phosphorylation of riboflavin (vitamin $\mathrm{B}_{2}$ ), in turn to produce the active forms of this vita$\min ^{35}$. Riboflavin has been found to act as a potential neuroprotective agent, preventing and/or modifying the process of neurological disorders by improving conditions of oxidative stress, mitochondrial dysfunction, and neuroinflammation ${ }^{36}$. Moreover, mRNA levels of human RFK (riboflavin kinase), the ortholog of yeast FMN1, have found to be significantly lower in the cerebellum, visual cortex and prefrontal cortex of AD patients versus controls (Fig. 2c).

Riboflavin biosynthesis in $S$. cerevisiae utilizes ribulose 5-phosphate and guanosine- $5^{\prime}$-triphosphate (GTP) as precursors and proceeds through pyrimidine intermediates ${ }^{37}$. The active forms of riboflavin, FMN and flavin adenine dinucleotide (FAD), are formed in two consecutive reactions which are catalyzed by FMN1 and FAD1 (FAD synthetase), respectively (Fig. 3a). The SGA screening showed that the fmn1 mutant was sensitive to A $\beta 42$ toxicity with a score of -0.55 and -0.66 from the two independent SGA screens. Moreover, complementation assays confirmed that $f m n 1$ mutant was crucial for $A \beta 42$ toxicity. As well, increased $\mathrm{A} \beta 42$ toxicity in the $f m n 1$ mutant background could be reduced by expression of FMN1 or the human ortholog RFK (Fig. 3b and Supplementary Fig. 8). We further measured the dynamic transcript levels of FMN1 in both $\mathrm{A} \beta 42$ and control strains. The results showed that FMN1 mRNA levels were significantly increased in the post-diauxic shift (PD) and stationary phases (SP) cells compared to exponentially growing cells (EX) in both $A \beta 42$ and control strains ( $p$-adj $<0.05$, Fig. $3 c)$. In the $\mathrm{A} \beta 42$ strain, there was $4.10,2.55,2.25$-fold increase in $\mathrm{PD}$, day 2 , and day 3 compared to EX, respectively. In the control strain, there was 4.4, 2.9, and 2.4-fold increase in PD, day 2, and day 3 compared to EX, respectively (Fig. 3c).

FMN supplementation reduces $\mathbf{A} \boldsymbol{\beta} 42$ toxicity. To test whether the increased $\mathrm{A} \beta 42$ toxicity was due to the fmn1 mutation or to a lack of downstream metabolic products, we cultivated the $\mathrm{A} \beta 42$ strain with $f m n 1$ mutation in culture medium containing either $5 \mathrm{mM}$ FMN or $5 \mathrm{mM} F A D$, a metabolic product that is one-step down from FMN in the riboflavin biosynthetic pathway. Here, the A $\beta 42$ toxicity was only found to be reduced by supplementation with $5 \mathrm{mM}$ FMN, not FAD (Fig. 3d). As well, supplementation with FMN could also rescue the growth impairment of the control strain with $f m n 1$ mutation (Supplementary Fig. 9). As a riboflavin-nucleotide coenzyme, FMN is essential for redox reactions and participates in a wide range of biological processes, including carbohydrate, fatty acid and amino acid metabolisms ${ }^{38}$. These observations suggest that maintaining a sufficient level of FMN may be required for both $\mathrm{A} \beta 42$ and control strains during different stages of cell growth. Furthermore, these results also suggest FMN deficiency could contribute towards A $\beta 42$-triggered toxicity. To test this, we evaluated cellular viability in the presence of different concentrations of FMN in the culture medium following chronological aging. The significantly increased viability of A $\beta 42$ strain was FMN-dosage dependent (Fig. 3e, f). The fractions of non-viable cells on day 5 were $61 \%$ in A $\beta 42$ strain without FMN, comparing to $45 \%, 39.4 \%$, 35\%, and $33.3 \%$ with $1,3,5$, and $7 \mathrm{mM}$ FMN added, respectively (Supplementary Fig. 10). Moreover, supplementation of FMN also improved viability in the control strain as well $(p$-adj $<0.05$, Supplementary Fig. 11).

FMN supplementation plays important roles in protein folding. Increasing evidence indicates that $\mathrm{A} \beta 42$ peptides are prone to form aggregates, which contributes notably to the neurotoxicity that occurs in $\mathrm{AD}$ pathogenesis ${ }^{39}$. To monitor whether the fmn1 mutation contributed to protein aggregation, we used GFP-tagged Hsp104 (heat shock protein 104) as a reporter for protein aggregation. In S. cerevisiae, Hsp104 plays a crucial role in thermotolerance, binding to aggregated proteins after exposure to 
a

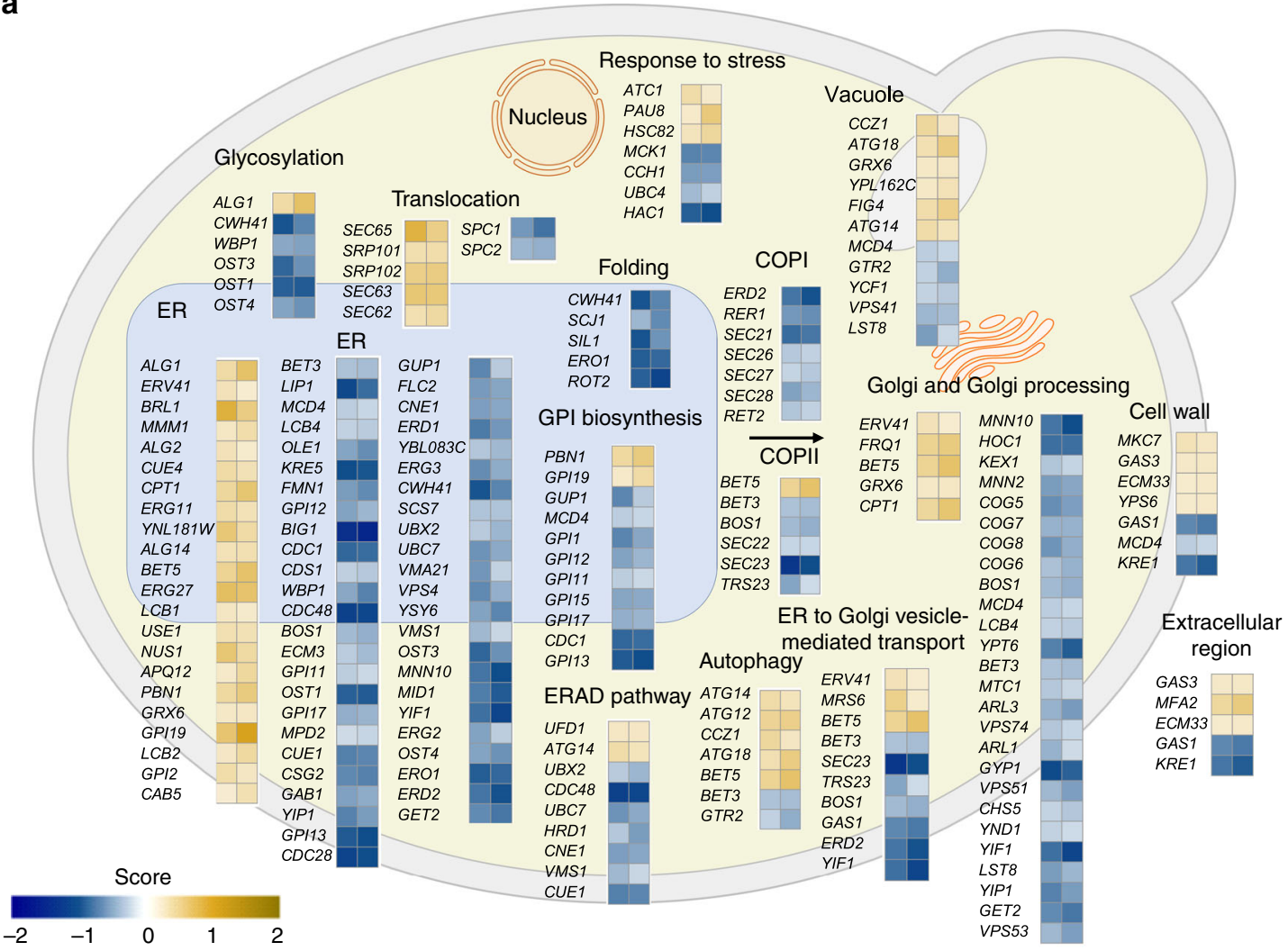

\begin{tabular}{|l|l|}
\hline Run 1 & Run 2 \\
\hline
\end{tabular}

b

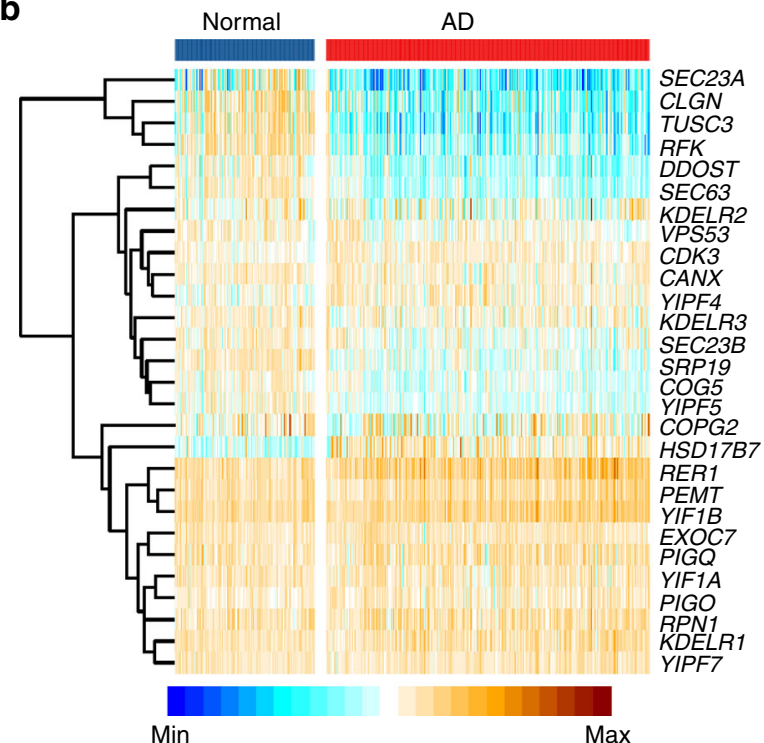

C

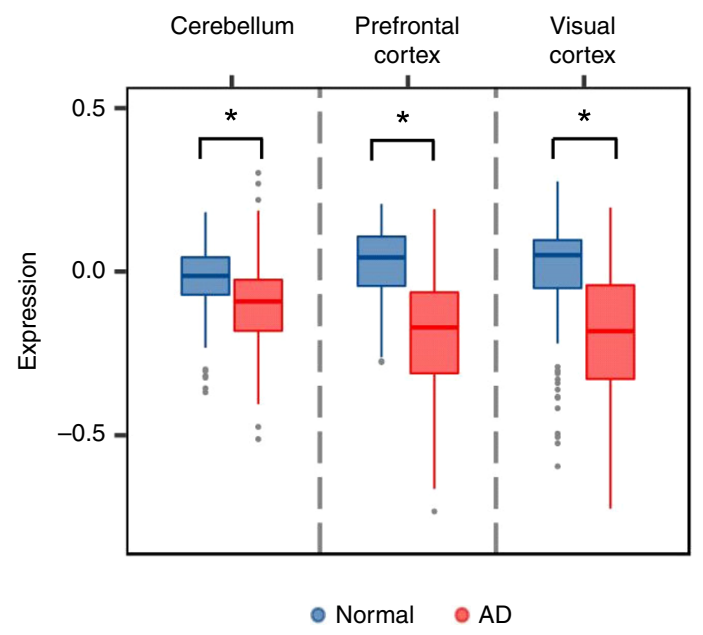

Fig. 2 Roles of protein secretion and degradation processes in A $\mathbf{\beta 4 2}$ toxicity. a Mutants with significantly changed A $\beta 42$ toxicity mapped to protein secretory and degradation processes ( $p$-adj $<0.05$ and score $\geq 0.2$ or $\leq-0.2$ ). b The mRNA expression levels of 28 human orthologs in the prefrontal cortex of Alzheimer's disease (AD) patients versus normal controls. Genes were ranked according to the size of their significant difference between the two groups. Out of the 28 human orthologs, 22 (79\%) showed significantly changed mRNA levels ( $p$-adj < 0.05). c Comparison of human RFK (ortholog of yeast FMN1) mRNA levels in three different brain regions (cerebellum, prefrontal cortex and visual cortex) between $A D$ patients and normal controls.

Asterisks $\left({ }^{*}\right)$ indicate significant differences ( $p$-adj $\left.<0.0001\right)$.

heat shock ${ }^{40}$. The GFP-tagged Hsp104 can therefore be observed as intracellular foci and inclusions using fluorescence microscopy ${ }^{41}$. Cells from both the $f m n 1$ mutant and the wild type were subjected to heat shock at $38^{\circ} \mathrm{C}$ for $90 \mathrm{~min}$ to induce protein misfolding and aggregation. Here, $f m n 1$ mutant was found to contain significantly $(p<0.001)$ more cells in the class 3 category $(55.5 \pm 4.6 \%, \geq 3$ aggregates/cell), than in the wild type strain $(10.4 \pm 5.7 \%$, Fig. $4 \mathrm{a})$. This indicates that FMN1 could be involved in sequestering small aggregates/oligomers into larger inclusion bodies, as part of a protective response towards reducing 
Table 1 Modifiers of Aß42 toxicity in protein secretion and degradation processes ${ }^{\mathrm{a}}$.

\begin{tabular}{llll} 
Systematic name & Standard name & Cellular function & Human ortholog \\
\hline YAL058W & CNE1 & Folding and quality control of glycoproteins & CANX/CLGN \\
YOR085W & OST3 & Oligosaccharyltransferase & TUSC3 \\
YDR236C & FMN1 & FMN biosynthesis & RFK \\
YEL002C & WBP1 & N-linked glycosylation of proteins in the ER & DDOST \\
YOR254C & SEC63 & Target and import proteins into ER & SEC63 \\
YBL040C & ERD2 & Maintenance of normal levels of ER-resident proteins & KDELR1/KDELR2/KEDLR3 \\
YGR172C & YIP1 & Biogenesis of ER-derived COPII transport vesicles & YIPF4/YIPF5/YIPF7 \\
YNL287W & SEC21 & ER to Golgi transport of selective cargo & COPG2 \\
YLR100W & ERG27 & Ergosterol biosynthesis & HSD17B7 \\
YCL001W & RER1 & Retention of membrane proteins to ER & RER1 \\
YGR216C & GPI1 & Synthesis of GlcNAc-PI & PIGQ \\
YLL031C & GPI13 & ER membrane localized phosphoryltransferase & PIGO \\
YJL002C & OST1 & Oligosaccharyltransferase & RPN1 \\
\hline aYeast genes with human orthologs were identified in SGA screening and confirmed by random spore test. & \\
\hline
\end{tabular}

proteotoxicity 42 . Correspondingly, we observed that $5 \mathrm{mM}$ FMN supplementation decreased $21 \%$ of aggregate formation in A $\beta 42$ strain, which links to A $\beta 42$ induced toxicity (Fig. $4 \mathrm{~b}$ ).

To further investigate if FMN supplementation can help cells to cope with a protein misfolding burden, we again stressed the cells, but this time directly targeted proteins as opposed to using general stress conditions. To achieve this, we used L-azetidine-2carboxylic acid (AZE), an analog of L-proline which causes protein misfolding. In the control strain, we observed that cellular viability was significantly $(p<0.05)$ decreased with $5 \mathrm{mM}$ and $10 \mathrm{mM}$ AZE treatment, but cells with $5 \mathrm{mM}$ FMN supplementation showed improved survival than without supplementation (Fig. 4c). Here, the number of viable cells was measured by colony forming units (CFU), which showed significantly $(p<0.05)$ higher viability in FMN supplemented cultures with either 5 or $10 \mathrm{mM}$ AZE treatment compared to cultures without FMN (Fig. 4d). This result indicates that FMN supplementation could increase cellular capacity to cope with misfolded protein stress.

FMN improves redox homeostasis in A $\beta 42$ strain. Oxidative stress has been implicated in many disease processes including neurodegenerative disorders and aging ${ }^{43}$. A balanced ratio of cellular oxidants and antioxidants is important to maintain a state of redox homeostasis, which is mainly determined by the ratios between reduced and oxidized forms of redox cofactors, particularly nicotinamide adenine dinucleotide phosphate (NADP) and nicotinamide adenine dinucleotide (NAD). In the A 342 strain, $5 \mathrm{mM}$ FMN supplementation resulted in a significant $(p<0.05)$ increase of the NADPH/(NADPH $\left.+\mathrm{NADP}^{+}\right)$ ratio during EX and PD conditions (Fig. 5a and Supplementary Fig. 12). Under these conditions, $\mathrm{NADH} /\left(\mathrm{NADH}+\mathrm{NAD}^{+}\right)$ ratios were also significantly $(p<0.05)$ improved as well (Fig. $5 \mathrm{~b}$ and Supplementary Fig. 12). Finally, FMN supplementation was also found to increase the $\mathrm{NADPH} /\left(\mathrm{NADPH}+\mathrm{NADP}^{+}\right)$ratio in the control strain without A $\beta 42$ expression $(p<0.05$, Supplementary Figs. 12 and 13a). As oxidative stress has been implicated as one of causative factors for aging ${ }^{44}$, the improved reducing environment might contribute to the increased viability in the control strain during chronological aging (Supplementary Fig. 11). Therefore, as well as lessening the propensity for protein misfolding, FMN supplementation may also alleviate A $\beta 42$ toxicity through improving redox homeostasis which can help overall cell response to oxidative stress.

To investigate this, we challenged the $\mathrm{A} \beta 42$ strain with the endogenous oxidant hydrogen peroxide $\left(\mathrm{H}_{2} \mathrm{O}_{2}\right)$. Here, cells were grown to mid exponential phase and exposed to incremental levels of $\mathrm{H}_{2} \mathrm{O}_{2}$. The length of the lag phase was calculated from exponential growth curves, by calculating the time after cells were arrested in growth following $\mathrm{H}_{2} \mathrm{O}_{2}$ treatment to the point where they returned to maximum growth rate ${ }^{45}$. We observed that $\mathrm{A} \beta 42$ cells displayed prolonged lag phases with higher $\mathrm{H}_{2} \mathrm{O}_{2}$ concentrations. However, the supplementation of $5 \mathrm{mM}$ FMN significantly $(p<0.05)$ shortened the extended lag phases (Fig. $5 c, d$ ). An oxidative stress tolerance assay for the control strain was also performed using spot tests with medium containing different concentrations of $\mathrm{H}_{2} \mathrm{O}_{2}$. As seen with the liquid growth assay, cells with $5 \mathrm{mM}$ FMN supplementation demonstrated a higher $\mathrm{H}_{2} \mathrm{O}_{2}$ tolerance than without supplementation (Supplementary Fig. 13b). Taken together, these results indicate that FMN supplementation can be beneficial for oxidative stress tolerance, in both $A \beta 42$ and control strains.

FMN supplementation causes a global transcriptional response. To gain further insight into the mechanism behind how FMN supplementation helps to improve cell physiology, A $\beta 42$ and control strains with and without $5 \mathrm{mM}$ FMN supplementation were sampled for RNA sequencing (Supplementary Fig. 12). Principal component analysis (PCA) showed that the A $\beta 42$ strain with FMN supplementation had a distinct gene expression profile (Supplementary Fig. 14a). Differential gene expression analysis identified more than a third of the genome ( $n=2081$ genes) to be significantly ( $p$-adj $<0.05$ ) up or downregulated in the A $\beta 42$ strain upon FMN supplementation (hereafter referred to as FMN_A $\beta 42$, Supplementary Data 3), compared to 389 genes in the control strain (hereafter referred to as FMN_control, Supplementary Fig. 14b and Supplementary Data 4). We next performed gene set analysis (GSA) on the significantly changed genes $(p$-adj $<0.05)$, wherein 53 and 22 gene sets were found to be significantly enriched in the FMN_A $\beta 42$ and FMN_control strain respectively $(p$-adj $<0.05$, Supplementary Fig. 15). Thirty genes were significantly differentially expressed in both FMN_A 342 and FMN_control $\left(p\right.$-adj $<0.05, \log _{2}$ Fold change $\leq-1$ or $\left.\geq 1\right)$, with 17 and 8 , respectively, out of these 30 genes being related to metabolic process and ion transport (Supplementary Fig. 16). This is consistent with the expected role of FMN as a cofactor for flavoproteins, participating in carbohydrate, amino acid, fatty acid and ion metabolic pathways ${ }^{46}$.

Gene set enrichment analysis also revealed that for the A $\beta 42$ strain with FMN supplementation, pathways that were upregulated were mainly involved in carbohydrate metabolism, 
a

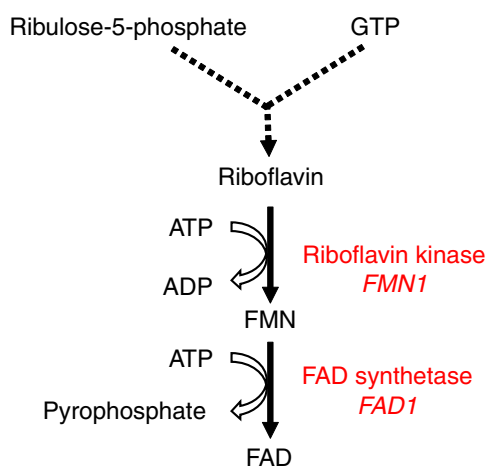

C

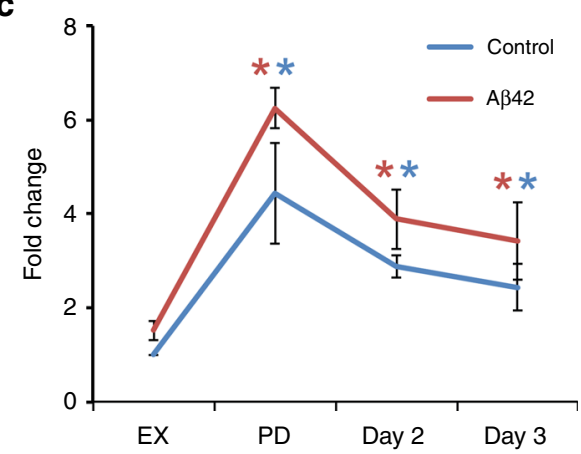

e

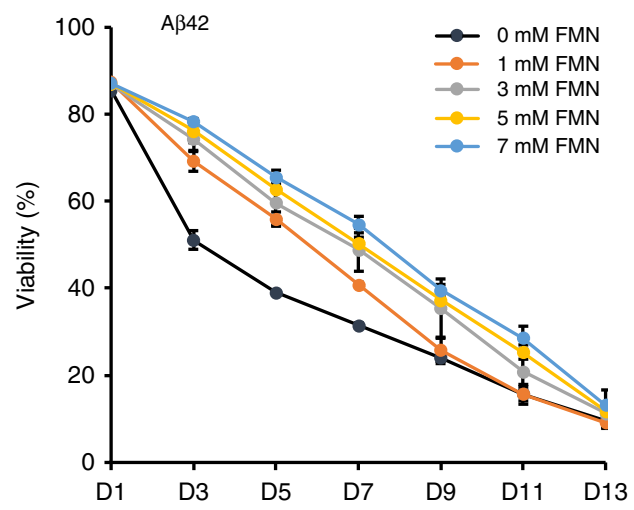

b

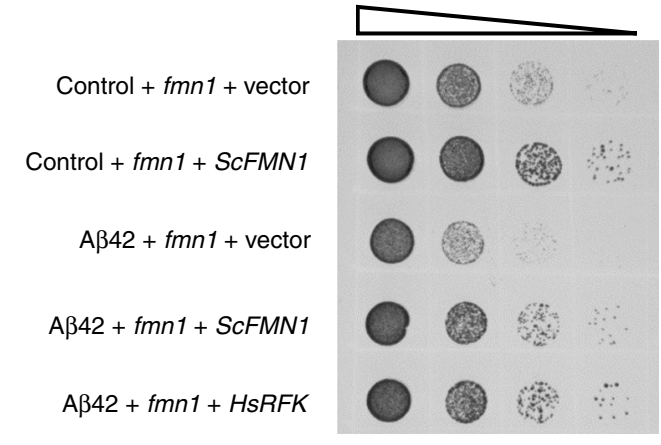

d

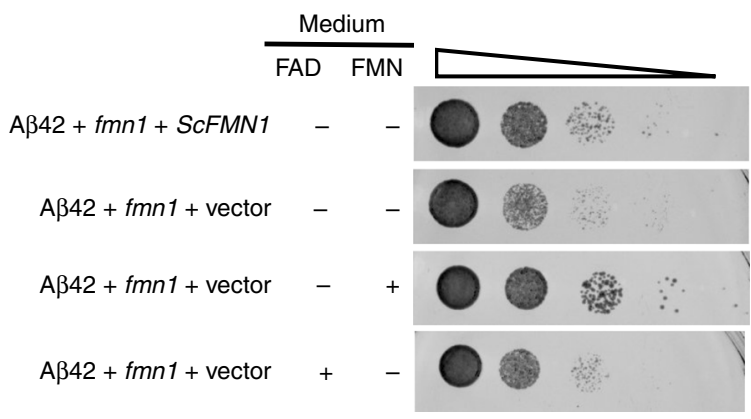

f

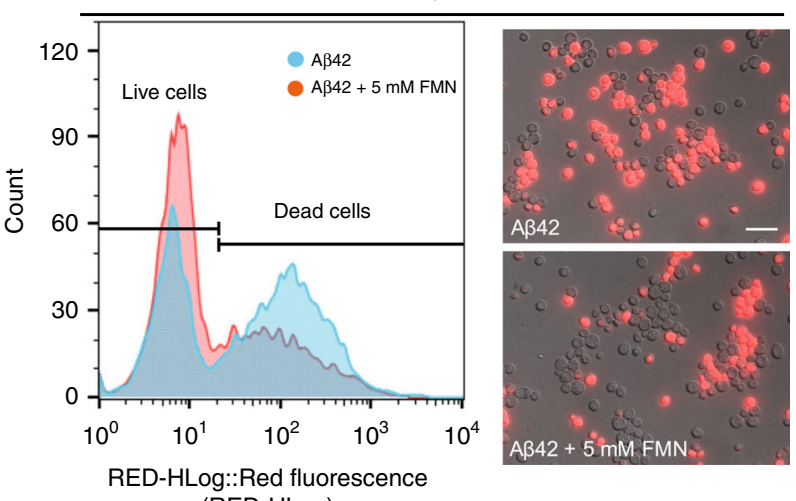

(RED-HLog)

Fig. 3 Riboflavin kinase and its metabolic product FMN reduce Aß42 toxicity. a Schematic illustration of the riboflavin biosynthesis metabolic pathway. FMN, flavin mononucleotide; FAD, flavin adenine dinucleotide. b Complementation assays to confirm fmn1 mutant is causal towards increasing A 442 sensitivity. Vector: MoBY empty plasmid; ScFMN1: pFMN1-MoBY plasmid; HsRFK: P416 GPD-human RFK plasmid. c FMN1 mRNA expression during exponential growth (EX), post-diauxic shift phase (PD) and stationary phase (day 2 and day 3). Results from qPCR are normalized to EX of each strain and shown as the average values \pm SD from three independent biological replicates. Asterisks $\left.{ }^{\star}{ }^{\star}\right)$ indicate significant differences compared to EX in both A $\beta 42$ (red) and control (blue) strains $(p<0.05)$. d Ten-fold serial dilutions of the A $\beta 42$ expression strain with fmn1 mutation carrying the aforementioned plasmids with supplementation of $5 \mathrm{mM} F A D$ or $5 \mathrm{mM} \mathrm{FMN}$ in the medium. e Survival of the A 442 strain during stationary phase with different concentrations of FMN supplemented. Viability is shown as the fraction of propidium iodide (PI) negative cells $(n=3 \pm$ SD). $\mathbf{f}$ Flow cytometry and fluorescence micrographs of PI staining in the A $\beta 42$ strain after 5 days of cultivation. Cells that are stained red represent nonviable cells. Scale $=10 \mu \mathrm{m}$. Source data are provided as a Source Data file.

glycogen biosynthesis, ion transport and homeostasis, ATP biosynthesis, mitochondrial translation, and cellular response to oxidative stress. In contrast, gene sets related to ribosome function, cytoplasmic translation, and cellular amino acid biosynthesis were all downregulated in the $\mathrm{A} \beta 42$ strain with FMN supplementation ( $p$-adj < 0.05 , Fig. $6 \mathrm{a}$ and Supplementary Fig. 15a). Many genes involved in amino acid biosynthesis were also significantly downregulated in the A $\beta 42$ strain with FMN supplementation ( $p$-adj $<0.05$, Supplementary Fig. 17). From these results one could speculate that FMN can improve protein homeostasis under stress by mediating the repression of amino acid biosynthesis and protein synthesis. This would enable a reduction in ER stress by reducing the load of newly synthesized proteins.

FMN supplementation significantly increased redox cofactors (Fig. 5a, b), that are mainly generated in carbohydrate metabolism $^{47}$, so we further investigated the differentially expressed genes in central carbon metabolism (Fig. 6b). $H X T 1^{48}$ and $H X T 3^{49}$, encoding low affinity glucose transporters, showed to be downregulated in the A $\beta 42$ strain with FMN supplementation. In contrast, genes encoding high affinity glucose 
a

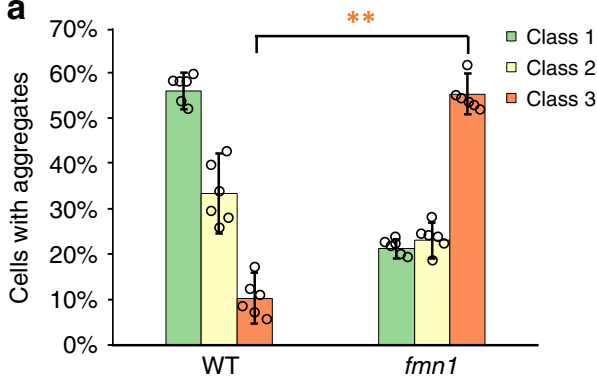

C

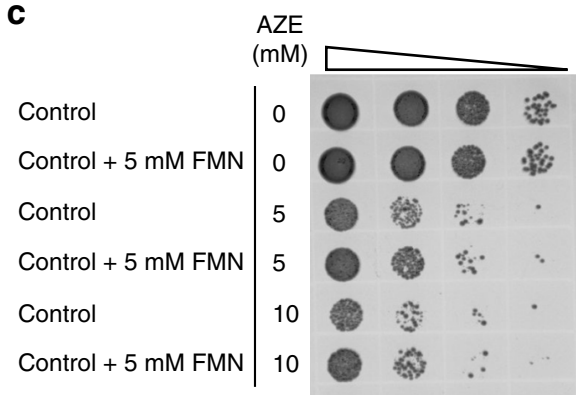

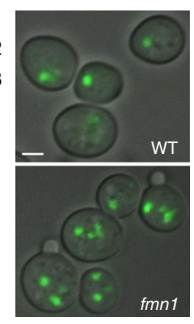

b

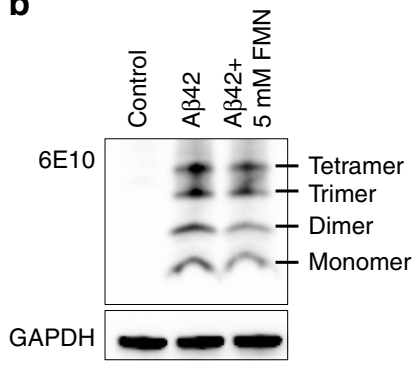

d

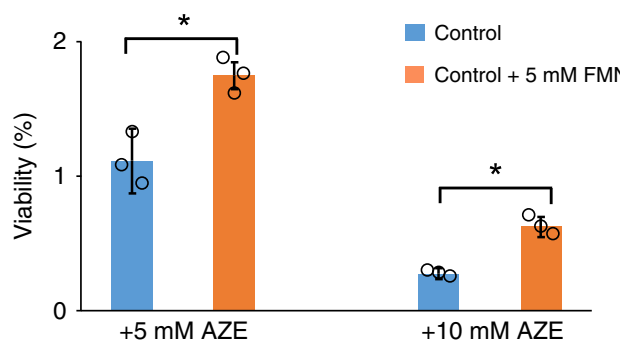

Fig. 4 FMN supplementation facilitates protein folding. a Quantification of aggregate foci after heat shock for 90 min in wild type and fmn1 mutant yeast cells. Class1: one aggregate per cell (as highlighted by single GFP cluster); Class 2: two aggregates per cell; Class 3: $\geq 3$ aggregates per cell. Bar graphs show fractions of Class 1, 2, and 3 cells for each strain. Above 500 cells were measured per sample with results shown as average values \pm SD from six biological experiments. Scale $=2 \mu \mathrm{m}$. ${ }^{\star \star} p<0.001$. b Western blot of A $\beta 42$ expression from stationary phase cultures, using the 6E10 A $\beta$ specific antibody. GAPDH was used as the loading control. c Ten-fold serial dilutions of the control strain after $3 \mathrm{~h}$ of treatment with 5 or $10 \mathrm{mM} \mathrm{L-azetidine-2-carboxylic} \mathrm{acid} \mathrm{(AZE).}$ d Colony forming unit (CFU) measurement of control strain after $5 \mathrm{mM}$ or $10 \mathrm{mM}$ of AZE treatment as a measurement of viability $(n=3 \pm \mathrm{SD})$. ${ }^{\star} p<0.05$. Source data are provided as a Source Data file.

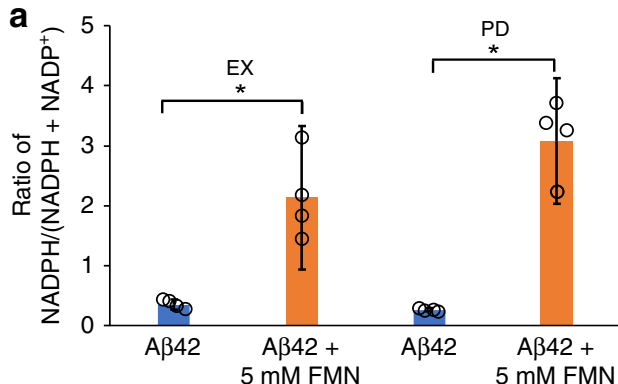

C
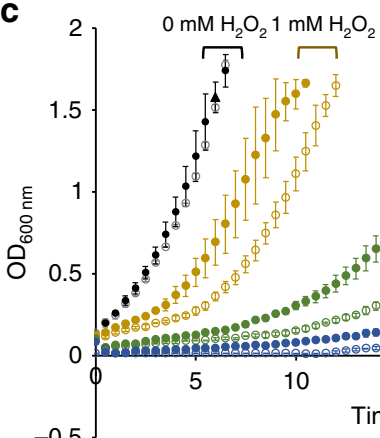

10

$-0.5$ b

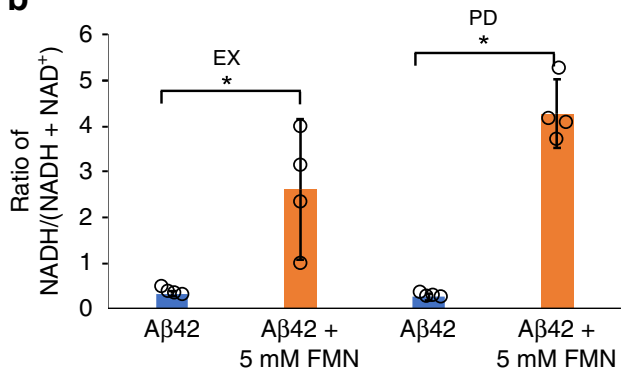

d

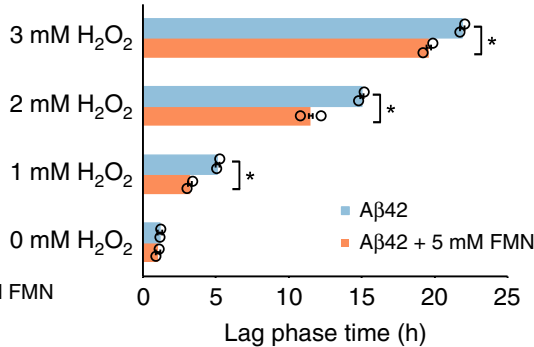

Fig. 5 FMN supplementation increases cellular tolerance to oxidative stress in $\mathbf{A} \beta 42$ strain. a Ratio of $N A D P H /(N A D P H+N A D P+)$ in the $A \beta 42$ strain without or with FMN supplementation during EX and PD growth phases respectively $\left(n=4 \pm \mathrm{SD} ;{ }^{\star} P<0.01\right)$. b Ratio of NADH/(NADH $\left.+\mathrm{NAD}+\right)$ in the $\mathrm{A} \beta 42$ strain without or with FMN supplementation during EX and PD growth phases $\left(n=4 \pm \mathrm{SD}\right.$; $\left.{ }^{\star} P<0.01\right)$. c, d A $\beta 42$ cells display an increased $\mathrm{H}_{2} \mathrm{O}_{2}$

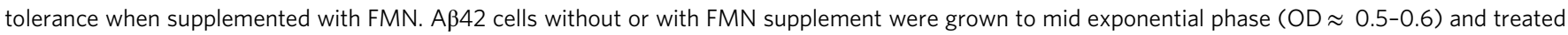
with different concentrations of $\mathrm{H}_{2} \mathrm{O}_{2}$. Cell growth was monitored using a microplate reader (c) with lag phase time analyzed using the $\mathrm{R}$ package growthrates (d). The lag phase indicates the time from $\mathrm{H}_{2} \mathrm{O}_{2}$ treatment until maximum growth rate, determined by the exponential growth phase. Results shown are average values \pm SD of biological duplicates. The asterisk $\left(^{\star}\right)$ indicates significant difference ( $p<0.05$ ). Source data are provided as a Source Data file. 
a

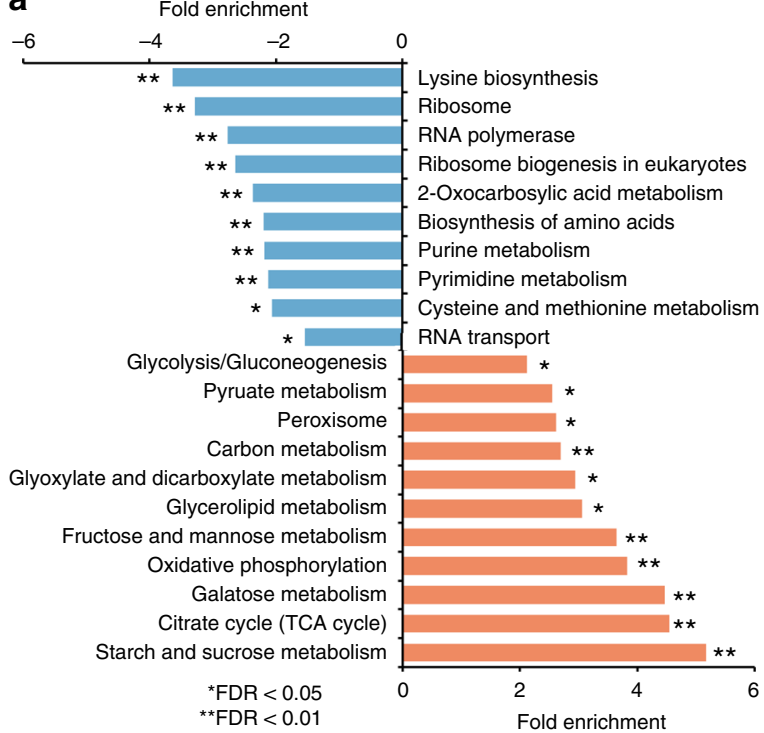

C

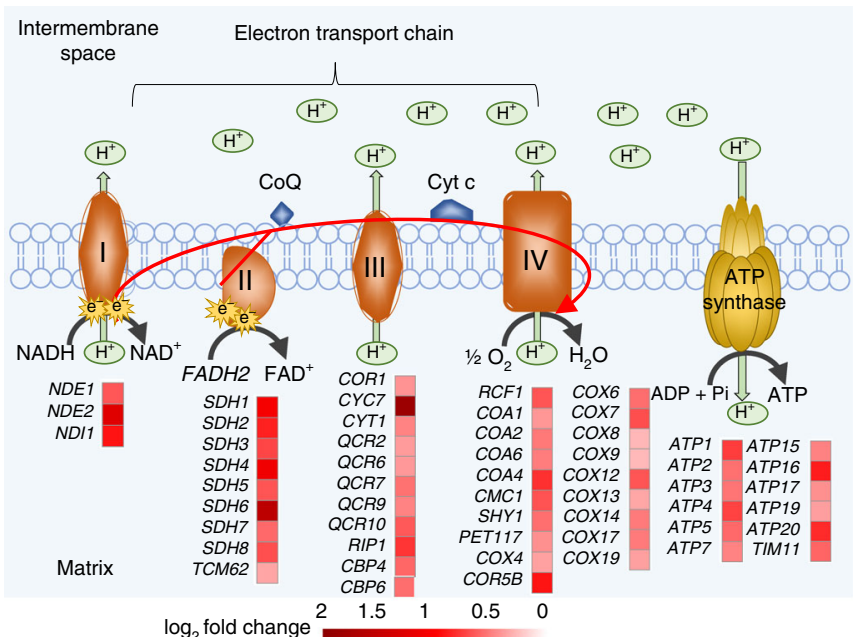

b

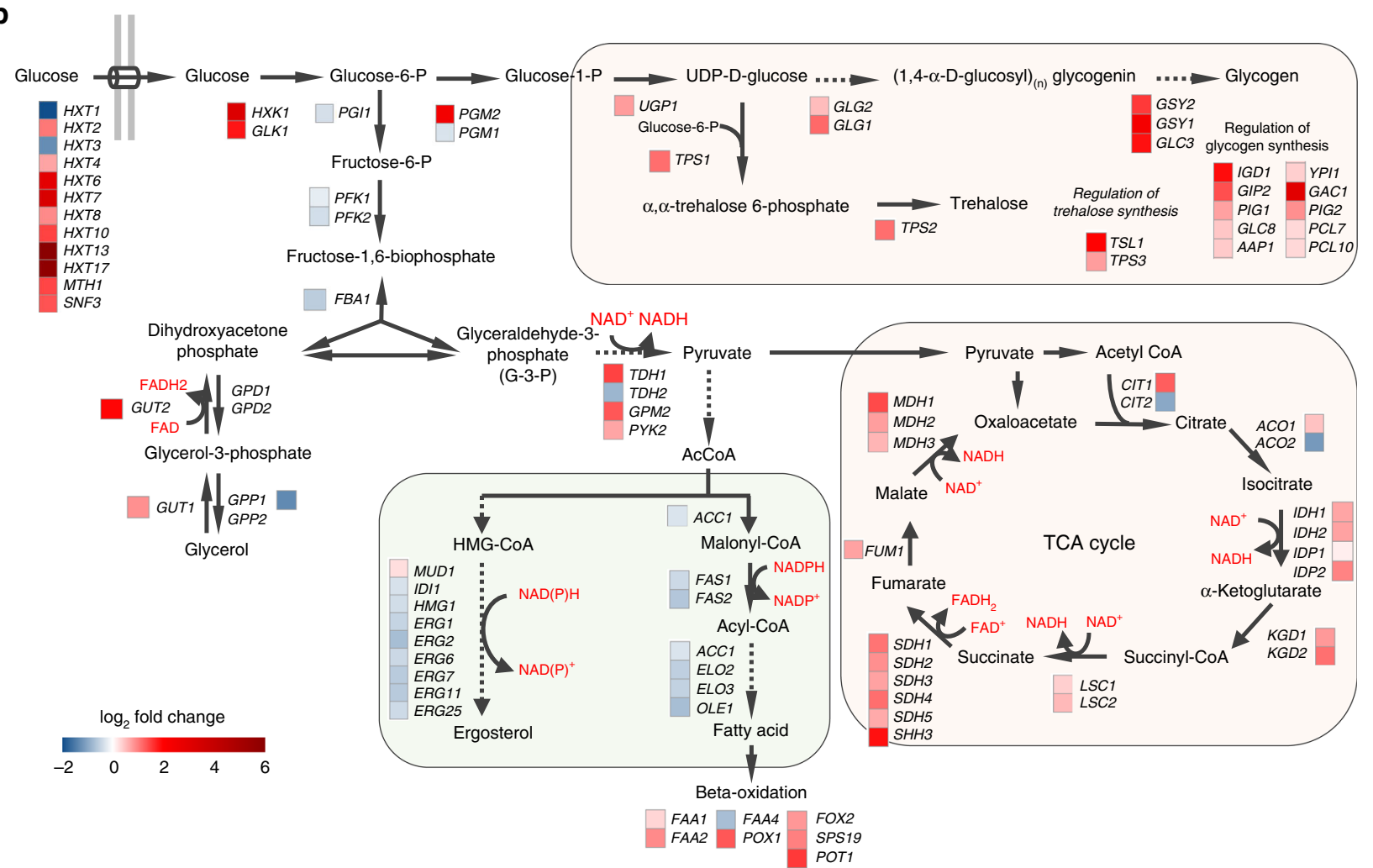

Fig. 6 Transcriptional responses in Aß42 strain with FMN supplementation. a Gene set enrichment analysis. Fold enrichment indicates the magnitude of enrichment in our dataset against the S288C yeast genome background using DAVID bioinformatics resources. $\mathbf{b}$ Fold changes in the expression of genes which encode central metabolic pathways. $\mathbf{c}$ Fold changes in expression of genes coding for mitochondrial electron transport chain proteins. All comparison is between FMN supplementation, and without ( $p$-adj $<0.05$ according to Benjamini-Hochberg method).

transporters (HXT2, HXT4, HXT6, and HXT7), and hexose transporters (HXT8, HXT10, HXT13, and HXT17) were upregulated. Most genes (20/22) in the tricarboxylic acid (TCA) cycle were also upregulated after FMN supplementation as well (Fig. 6b). The TCA cycle generates $\mathrm{NADH}$ and $\mathrm{FADH}_{2}$, which in turn act as electron donors for oxidative phosphorylation in the electron transport chain. Meanwhile, we found that genes encoding respiratory complexes in the electron transport chain showed significantly $(p<0.05)$ increased transcription after FMN supplementation (Fig. 6c). Iron transport plays a vital role in living organisms by participating in a variety of electron transfer reactions and enzymatic reactions. Here, genes involved in iron transport and homeostasis were also mostly upregulated after FMN supplementation (Supplementary Fig. 18). In terms of what decreased in expression after FMN supplementation, genes involved in ergosterol and fatty acid synthesis, the second branch of pyruvate metabolism, were mainly downregulated, along with a reduced NADPH consumption to $\mathrm{NADP}^{+}$. This finding is 
consistent with the increased $\mathrm{NADPH} /\left(\mathrm{NADPH}+\mathrm{NADP}^{+}\right)$ ratio we found in the FMN supplemented strains (Fig. 5a). The synthesis pathways for the storage carbohydrates glycogen and trehalose were also enriched with upregulated genes after FMN supplementation (Fig. 6b). The upregulated genes in trehalose synthesis may therefore link the stress resistant phenotype of FMN supplemented cells to the alternative role of trehalose as a stress protectant. Indeed, it has been reported previously that trehalose can stabilize the structures and enzymatic activities of proteins against thermal denaturation ${ }^{50}$, and can prevent the formation of $A \beta$ aggregates and reduce its cytotoxicity ${ }^{51}$.

Our previous observations showed that $A \beta 42$ expression induces strong ER stress and activates the UPR as a means of the cell attempting to reduce the misfolded protein burden generated by $\mathrm{A} \beta 42^{23}$. After FMN supplementation of the A $\beta 42$ strain, genes related to the oxidative stress response, protein degradation, and endocytosis were found to be significantly $(p<0.05)$ upregulated (Supplementary Fig. 19), suggesting cells are restoring ER homeostasis. These findings are consistent with western blot analysis showing that FMN supplementation decreased levels of A $\beta 42$ aggregates (Fig. 4b).

Overexpression of flavoprotein Dus2p reduces $A \beta 42$ toxicity. FMN participates in many biological processes, as can be seen through our transcriptional analysis following FMN supplementation. This could principally be due to its role as an essential cofactor for a variety of FMN-dependent enzymes. To investigate the potential roles of flavoenzymes in general in A $\beta 42$ toxicity, 11 strains with single flavoenzyme gene deletions were assessed. These flavoenzymes are involved in myriad roles, from reactions involving electron transfer in the mitochondria (Cyb2p, Glt1p, Pst2p), redox processes in the cytoplasm (Lot6p, Oye2p, Rfs1p), tRNA-modification (Dus2p, Dus3p, Dus4p), to central metabolic functions (Met5p, Aro2p) ${ }^{52}$. First, the 11 single deletion strains were transformed with a plasmid for constitutive expression of A $\beta 42$. Thereafter, cellular viability during chronological aging was tested with $5 \mathrm{mM}$ FMN supplementation. As found previously (Fig. 3e), we observed that FMN significantly increased A $\beta 42$ survival. As in several strains, despite flavoenzyme genes being deleted, FMN supplementation was still found to significantly increase $\mathrm{A} \beta 42$ viability in the mutant strains: $r f s 1 \Delta$, pst $2 \Delta$, lot $6 \Delta$, oye $2 \Delta$, aro $2 \Delta, d u s 3 \Delta$ and $d u s 2 \Delta(p<0.05)$. FMN supplementation however did not affect $\mathrm{A} \beta 42$ viability in $d u s 4 \Delta$, $c y b 2 \Delta$ and glt1 $\Delta$ mutant strains $(p>0.05)$. Finally, in contrast, FMN supplementation significantly decreased $A \beta 42$ viability in the met5 $\Delta$ mutant strain ( $p<0.05$, Supplementary Fig. 20a).

Since $c y b 2 \Delta, g l t 1 \Delta$, and met5 $\Delta$ significantly altered the beneficial effects of FMN supplementation in the $A \beta 42$ strain, we tested to see if the opposite would be true if $A \beta 42$ toxicity could be alleviated by overexpression of these genes. Except for $c y b 2$, glt 1 and met5 genes, dus 2 gene was also tested due to dus $2 \Delta$ significantly decreased $\mathrm{A} \beta 42$ viability $(p<0.05,9.2 \pm 0.9 \%$ of live cells in dus $2 \Delta$ strain vs $89.3 \pm 0.9 \%$ of the control A $\beta 42$ strain). Only DUS2 overexpression significantly increased viability in A $\beta 42$ strain, showing $71.4 \pm 4.5 \%$ and $44.3 \pm 5.2 \%$ of live cells, in 1 - and 4-day-old stationary phase cultures respectively, compared to $60.8 \pm 5.5 \%$ and $31.1 \pm 3.4 \%$ of the control $A \beta 42$ strain $(p<$ 0.05 , Supplementary Fig. 20b). This would therefore suggest that DUS 2 could be involved in modulating A $\beta 42$ toxicity. The mRNA expression levels of human ortholog DUS2 were also significantly lower in cerebellum, visual cortex and prefrontal cortex of $\mathrm{AD}$ patients, compared to controls (Supplementary Fig. 21). Moreover it has been reported that Dus2 serves as an inhibitor in the regulation of $\mathrm{PKR}$ function ${ }^{53}$, an interferon-induced protein kinase. As the activation of PKR is known to be related to ER stress-induced apoptosis in $\mathrm{AD}^{54}$, DUS2 could be investigated as an additional gene target alongside FMN1 for reducing $\mathrm{A} \beta 42$ toxicity.

FMN reduces HTT103QP and a-synuclein toxicity. Many neurodegenerative disorders share a common hallmark of aberrant protein aggregation. To test the specificity of FMN effects, we assessed its effect on two other proteins: HTT103QP and a-synuclein, using humanized yeast models.

HD develops when the CAG trinucleotide repeats cause the polyglutamine (polyQ) expansion in the Huntingtin (HTT) gene $^{55}$. In yeast, repeat lengths of 47 or more lead to HTT protein aggregation and the formation of typical inclusion bodies that are similar to those of HD patients ${ }^{56}$. The HTT103QP model was reconstructed with 103 glutamines expansion under the control of constitutive GPD promoter. We then evaluated cellular viability in the presence of $5 \mathrm{mM}$ FMN following chronological aging. The fractions of viable cells were significantly higher until day 11 in the HTT103QP strain with FMN supplementation, compared to without supplementation (Fig. 7a). In previous experiments, we observed that FMN supplementation was beneficial for oxidative stress tolerance in A $\beta 42$ strain. Since increased oxidative stress has long been held as a key player in the HD pathogenesis ${ }^{57}$, we further investigated if FMN supplementation could protect HTT103QP cells from oxidative stress, as was the case in A $\beta 42$ strain. The results showed that HTT103QP cells had prolonged lag phases during $\mathrm{H}_{2} \mathrm{O}_{2}$ stress, and the FMN supplementation could significantly shorten the extended lag phases $(p<0.05$, Fig. $7 c$ and Supplementary Fig. 22a).

The pathological hallmark of PD is the accumulation of high levels of oxidative free radicals and the presence of cytoplasmic inclusions called Lewy bodies, which predominantly composed of the misfolded and aggregated $\alpha$-synuclein ${ }^{58}$. In the humanized PD yeast model, the expression of $a$-synuclein was controlled under the inducible MET25 promoter, which is induced by depletion of methionine from the culture medium ${ }^{59}$. We observed that supplementation of FMN improved cellular viability in the $a$-synuclein strain as well $(p<0.05$, Fig. $7 b)$. The oxidative stress tolerance assay was also performed, which showed cells with FMN supplementation had a higher $\mathrm{H}_{2} \mathrm{O}_{2}$ tolerance than cultures without supplementation $(p<0.05$, Fig. $7 \mathrm{~d}$ and Supplementary Fig. 22b). These results indicate that FMN supplementation could offer increased oxidative stress tolerance in different neurodegenerative disease models.

\section{Discussion}

In this study, we used genome-wide SGA screening to identify modulators of A $\beta 42$ cytotoxicity. From nearly 5,500 mutants we identified a subset with increased sensitivity towards $A \beta 42$, which were significantly enriched in protein secretion and degradation processes, as well as to cytoprotective mechanisms for maintaining cellular proteostasis. Moreover, we specifically identified riboflavin kinase (FMN1 gene), and its metabolic product FMN, as critical modulators of $A \beta 42$ toxicity. Transcription of $R F K$, the human orthologue of FMN1, was also found to be significantly decreased in brain tissues of $\mathrm{AD}$ patients (Fig. 2c), thereby suggesting a conserved evolutionary function of riboflavin kinase in underlying processes that govern proteostasis management in cells.

Brain cells are particularly susceptible to oxidative stress due to their higher metabolic activity and lower antioxidant activity, compared to many cells of other organs, suggesting that redox metabolism may be an important factor for neuronal death. There are several plausible models proposing how riboflavin could protect cells against oxidative stress by: (1) enhancing the 
a

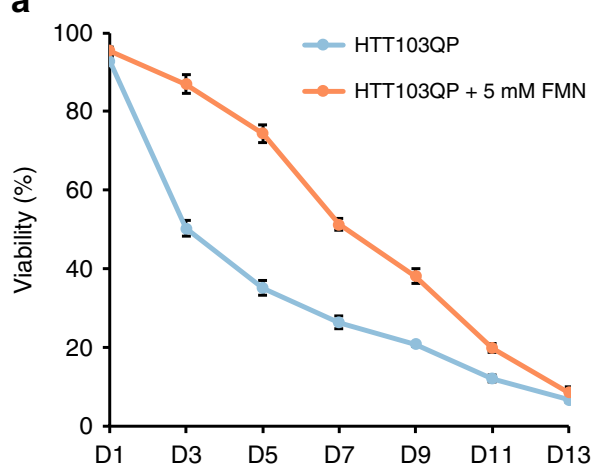

C

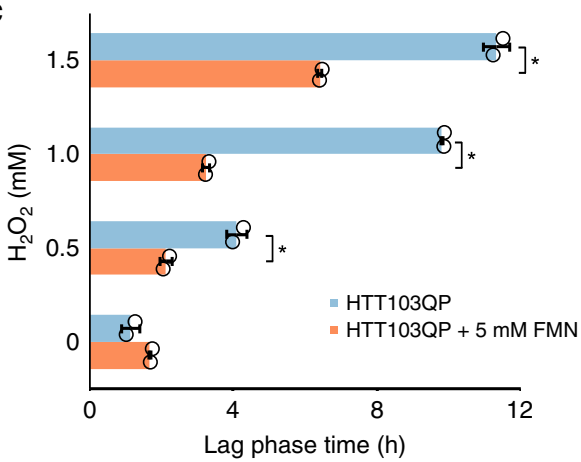

b

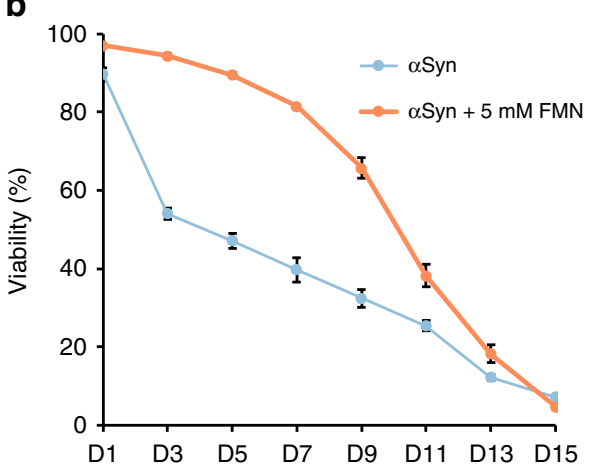

d

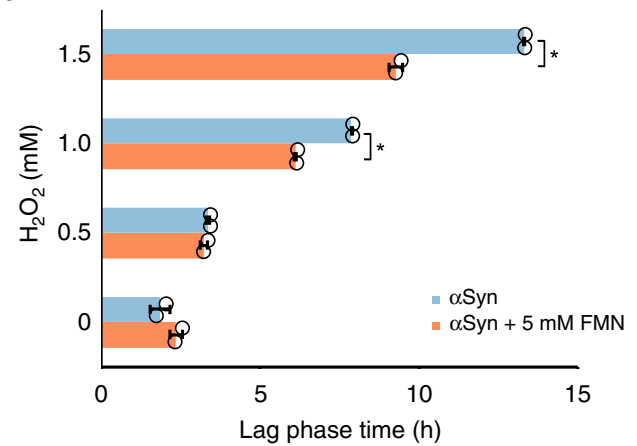

Fig. 7 FMN supplementation reduces HTT103QP and $\boldsymbol{\alpha}$-synuclein toxicity. Viability of the HTT103QP strain (a) and the $\alpha$-synuclein strain (b) with 5 mM FMN supplemented during chronological aging. Viability is shown as the fraction of PI negative cells $(n=3 \pm$ SD). HTT103QP cells (c) and $\alpha$-synuclein cells (d) display an increased $\mathrm{H}_{2} \mathrm{O}_{2}$ tolerance with $\mathrm{FMN}$ supplementation. Cells without or with $\mathrm{FMN}$ supplement were grown to OD $\approx 0.2$ and treated with different concentrations of $\mathrm{H}_{2} \mathrm{O}_{2}$. Cell growth was monitored using a microplate reader with lag phase time analyzed using the $\mathrm{R}$ package growthrates. Results shown are average values \pm SD of biological duplicates. The asterisk $\left({ }^{\star}\right)$ indicates significant difference $(p<0.05)$. Source data are provided as a Source Data file.

glutathione redox cycle ${ }^{60}$, (2) deactivating the lipid peroxides ${ }^{61}$, (3) direct activity against free radicals ${ }^{62}$, and (4) enhancing antioxidant enzyme activities ${ }^{63}$. Oxidative stress is also often accompanied by mitochondrial dysfunction, which may exacerbate disease progression ${ }^{64}$ and it was shown in several case studies that riboflavin administration in patients with mitochondrial diseases can lead to clinical improvement ${ }^{65,66}$.

The crucial roles of riboflavin are linked to its active forms FMN and FAD. With a tricyclic heteroaromatic isoalloxazine ring, FMN and FAD can reversibly accept and donate one or two electrons, with the majority of flavoenzymes catalyzing reductionoxidation reactions in metabolic pathways. Transcriptomics analysis of our yeast $\mathrm{AD}$ model showed that the genes involved in TCA cycle and oxidative phosphorylation processes were significantly upregulated by FMN supplementation (Fig. 6), suggesting that FMN mediates an increase in the generation of ATP and NADH. In addition, we also observed significantly downregulated expression of genes related to ergosterol and fatty acid synthesis pathways, indicating possible increase in the release of reduced NADPH (Fig. 6).

Declining mitochondrial function is known to lead to abnormal energy metabolism, which plays an important role in mediating $\mathrm{AD}$ dysfunction and degeneration ${ }^{67,68}$. Mitochondrial dysfunction also often accompanies elevated ROS production, leading to oxidative stress that may exacerbate $\mathrm{AD}$ progression through oxidative damage to cellular structures, proteins, lipids and $\mathrm{DNA}^{64}$. In our previous report, aberrant fragmented mitochondrial structures and increased ROS levels in our A $\beta 42$ model were indeed observed ${ }^{23}$. The redox couples $\mathrm{NAD}(\mathrm{P}) \mathrm{H} / \mathrm{NAD}(\mathrm{P})^{+}$ are the major electron transfer carriers for reduction-oxidation in cells, and thus vital for diverse biochemical processes. During aging, decreased $\mathrm{NAD}(\mathrm{P}) \mathrm{H}$ and redox ratios could contribute to $\mathrm{AD}$ progression, which may be due to a decline in TCA cycle enzymes activites ${ }^{69}$. Studies in aging and $\mathrm{AD}$-like neurons from nontransgenic and $3 \times \mathrm{Tg}-\mathrm{AD}$ mice showed that adding an NAD $(\mathrm{H})$ precursor, in order to stimulate a balanced redox defense is neuroprotective ${ }^{70}$. In accordance with previous findings, we observed significantly increased $\mathrm{NADPH} /\left(\mathrm{NADPH}+\mathrm{NADP}^{+}\right)$ and $\mathrm{NADH} /\left(\mathrm{NADH}+\mathrm{NAD}^{+}\right)$ratios and cellular tolerance to oxidative stress in our $A \beta 42$ strain when supplemented with FMN (Fig. 5), which may partially contribute to the increased viability during chronological aging (Fig. 3e, f and Supplementary Fig. 11). Targeting redox metabolism and FMN cofactors may thereof be an effective therapy for combating oxidative stress in aging and $\mathrm{AD}$.

Another important group of flavoenzymes are involved in reactions related to tRNA-modifications. In particular, the Dus1-4 family of FMN-dependent flavoenzymes, encode tRNAdihydrouridine synthases, which reduce uracil to dihydrouridine at specific positions on tRNA. Moreover, this is one of the most common modifications of nucleosides in tRNA in all living cells $^{71}$. So far, only one dihydrouridine synthase in human has been identified, which is an ortholog of the yeast Dus $2 \mathrm{p}^{72}$. The human Dus2 $p$ has also been found to be upregulated in pulmonary carcinogenesis patients, resulting in higher levels of dihydrouridine modification ${ }^{73}$. In our $A \beta 42$ strain, dus $2 \Delta$ significantly decreased cellular viability (Supplementary Fig. 20a), whilst DUS2 overexpression significantly increased viability, suggesting that Dus2p might be a direct contributing factor towards A $\beta 42$ detoxification (Supplementary Fig. 20b). Further 
investigation on the biological significance of Dus2 could improve our understanding of this flavoprotein in $\mathrm{AD}$ pathogenesis as well as help determine its potential as a therapeutic target against the disease.

With respect to the fmn1 mutant identified from SGA screening, the lack of the functional enzyme resulted in the formation of multiple amorphous-like aggregates, rather than one or two large inclusions (Fig. 4a). Previous work has found these amorphous aggregates to be severely toxic to yeast cells ${ }^{74}$, however, supplementation of FMN, the product of FMN1 activity, helped alleviate misfolded protein stress in control strain (Fig. 4c, d).

In $\mathrm{AD}$, ER stress plays one of the principle roles in determining disease pathogenesis, as it leads to activation of the UPR, which aims to restore ER homeostasis. Previous findings show that UPR signaling can be modulated through finetuning the expression of various gene targets, depending on the nature of the stress ${ }^{75}$. In our previous study we found that $A \beta 40$ triggers a mild stress response, whilst $A \beta 42$ triggers a significantly stronger stress response, alongside a stronger UPR response, affecting many aspects of cell physiology 23 . Considering the global effects of $\mathrm{A} \beta 42$, we used genome-wide transcriptional analysis to understand how $A \beta 42$ toxicity is alleviated by FMN supplementation. FMN showed to enhance the efficacy of UPR in the A $\beta 42$ strain by downregulating amino acid synthesis and upregulating protein degradation (Supplementary Fig. 17 and 19). This may also explain our findings that $A \beta 42$ aggregates in our $A \beta 42$ strain were reduced following supplementation with FMN (Fig. 4b).

In the ER, the relative higher concentration of oxidized glutathione (GSSG) is essential for the formation of native disulphide bonds in nascent proteins ${ }^{76}$. Reduced glutathione (GSH) also contributes towards robust cell function by acting as an endogenous antioxidant and deactivating ROS. To control ER redox homeostasis during protein folding, oxidized GSSG should therefore be reduced to GSH through glutathione reductase, which requires $\mathrm{FAD}$ that is produced from FMN, as a coen$z^{2 y m e}{ }^{77}$. The connection between the glutathione cycle and its dependency on flavins in the ER, may therefore also contribute to the beneficial effects of FMN supplementation.

Genome-wide screenings have been performed in yeast models with a mutant HTT fragment or $\alpha$-synuclein, which showed the toxicity medicated by these two proteins was regulated by different sets of conserved genes and pathways ${ }^{78}$. Genes that modify the mutant HTT fragment cluster in the cellular processes related to stress response, protein folding, and ubiquitin-dependent protein catabolism, whereas genes in the functional categories of lipid metabolism and vesicle-mediated transport processes are isolated in the $\alpha$-synuclein screen ${ }^{78}$. A yeast genome-wide screen based on high content microscopy (HCM) found that huntingtin exon 1 (Httex1)-103Q expression showed the ribosome quality control (RQC) machinery as playing a key role in regulating the nuclear localization and adverse effects of Httex1-103Q proteins ${ }^{79}$. Although various biological processes were involved in $\mathrm{AD}, \mathrm{HD}$ and $\mathrm{PD}$, the oxidative stress is critical to neuronal death in neurological disorders ${ }^{80}$. Neurons are particularly vulnerable to oxidative damage due to their high oxygen consumption $(20 \%$ of total consumption), abundance of peroxidation-susceptible lipid bilayer and weak antioxidant defense ${ }^{80}$. The beneficial effects of FMN for oxidative stress tolerance in $\mathrm{AD}, \mathrm{PD}$ and $\mathrm{HD}$ yeast models showed potential to further explore the effectiveness in attenuating neurodegenerative pathology.

In summary, our $A \beta$ yeast model provides a useful tool for identifying genetic modifiers of $A \beta$ cytotoxicity and investigating their mechanisms of action. Furthermore, this study provides highly informative data regarding the beneficial effects of FMN supplementation on cellular metabolic activity, redox homeostasis and proteostasis in the $A \beta 42$ strain. Due to a strong correlation between oxidative stress, metabolic syndrome and $\mathrm{AD}^{81}$, understanding how FMN and flavoenzymes attenuate $A \beta$-induced cytotoxicity could be highly beneficial for clarifying molecular mechanisms in $\mathrm{AD}$ onset and progression.

\section{Methods}

Plasmids and strains. All plasmids and yeast strains used in this study are specified in Supplementary Tables 5 and Table 6, respectively. The primers used in this study are listed in Supplementary Table 7. E. coli DH5a was used for plasmid amplification. Plasmids for gene overexpression were constructed by Gibson assembly of the gene fragments, which were amplified from CEN.PK 113-11C genomic DNA, with a p416 GPD vector backbone, resulting in plasmids p416 GPD-FMN1, p416 GPD-CYB2, p416 GPD-GLT1, p416 GPD-MET5 and p416 GPD-DUS2, respectively. Codon optimized human RFK gene was synthesized and digested with restriction enzymes and ligated into the p416 GPD plasmid. The Molecular Barcoded Yeast (MoBY) plasmids and empty vector control (p5586) were kindly provided by the Department of Chemistry \& Molecular Biology, Gothenburg university ${ }^{82}$. Genomic integration was done by all-in-one plasmids pECAS9-gRNA-KanMX-XI-3 and pECAS9-gRNA-KanMX-XII-5, which express high-fidelity version of Cas9 nuclease and guide RNA for XI-3 and XII-5 site respectively ${ }^{83}$. The Cas 9 cassette and vector backbone were amplified from pECAS9-gRNA-KanMX-tHFD1 plasmid using primers CAS9-L1/CAS9-R1 and CAS9-L2/CAS9-R2, respectively. The specific guide RNAs were selected by comparing all potential off-targets in the CEN.PK 113-7D genome using the online CRISPRdirect tool (http://crispr.dbcls.jp/) ${ }^{84}$. The pECAS9-gRNA-KanMX-XI-3 and pECAS9-gRNA-KanMX-XII-5 plasmids were generated by Gibson Assembly Master Mix (New England BioLabs). The pYES2 vector expressing GFP-tagged HTT103QP construct under the GAL1 promoter was described previously ${ }^{85}$. The HTT103QP and GFP fragment were amplified and assembled into p416 GPD vector backbone by Gibson assembly, resulting in p416 GPD-HTT103QP plasmid. The pUG23 a-syn plasmid expressing a full-length $\alpha$-synuclein-EGFP sequence was kindly provided by Prof. Joris Winderickx, KU Leuven, Belgium ${ }^{59}$. The standard LiAC/SS carrier DNA/PEG method was used for yeast transformation ${ }^{86}$. Control and $A \beta 42$ strains were query strains for SGA screening, which were constructed through homologous recombination by co-transformation of a repair fragment and pECA9-gRNA plasmid to Y7092 strain (Supplementary Fig. 1). Two repair fragments, XI-3up + (GPDp-A $342-C Y C 1 t)+($ TEF1p-natNT2-ADH1t $)+$ XI-3down and XII-5up + (GPDp-Aß42-CYC1t $)+($ TEF1p-hphNT1-ADH1t $)+$ XII-5down, were prepared for two copies of $A \beta 42$ gene integration. The upstream and downstream homologous arms XI-3up and XI-3down were amplified from Y7092 genomic DNA with primers XI-3-up-L1/ XI-3-up-R1 and XI-3-down-L/ XI3-down-R, respectively. The GPDp-A $342-C Y C 1 t, T E F 1 p-n a t N T 2-A D H 1 t$ and TEF1p-hphNT1-ADH1t cassettes were amplified from p416 GPD-A 342 , pFA6anatNT2 and pFA6a-hphNT1 plasmids, respectively. The upstream and downstream homologous arms XII-5up and XII-5down were amplified from Y7092 genomic DNA with primers XII-5-up-L/XII-5-up-R and XII-5-down-L2/ XII-5down-R, respectively. The repair fragments were assembled by fusing the different DNA parts with overlapping PCR. For the control strain, the GPDp-CYC1t cassette was amplified from $\mathrm{p} 416 \mathrm{GPD}$ plasmid. Transformants were selected on YPD plate supplemented with $100 \mathrm{mg} \mathrm{L}^{-1}$ clonNat (Werner BioAgents) and $300 \mathrm{mg} \mathrm{L}^{-1}$ hygromycin $\mathrm{B}$ (Formedium). Control (CEN) and $\mathrm{A} \beta 42(\mathrm{CEN})$ strains used for verification were constructed in CEN.PK 113-11 C background following the same method. The homologous recombination-based method was used for the construction of a fmn1 Hsp104-GFP strain. The Hsp104-GFP-Leu fragment along with $500 \mathrm{bp}$ upstream sequence and $600 \mathrm{bp}$ downstream sequence was amplified from WT Hsp104-GFP strain (BY4741 background) genomic DNA and transformed into fmn1 mutant strain to replace endogenous HSP104 sequence. SD Leu plates containing $200 \mathrm{mg} \mathrm{L}^{-1} \mathrm{G} 418$ (Formedium) were used for selection of transformants at $22^{\circ} \mathrm{C}$.

Culture conditions. E. coli was grown in LB medium supplemented with $100 \mathrm{mg} \mathrm{L}^{-1}$ ampicillin or $50 \mathrm{mg} \mathrm{L}^{-1}$ kanamycin at $37^{\circ} \mathrm{C}$. Yeast strains were grown in YPD medium, Delft medium, SD-Ura medium or SD-Leu medium according to the auxotrophic and antibiotic phenotypes of the cells (Supplementary Table 6). YPD medium contained $10 \mathrm{~g} \mathrm{~L}^{-1}$ yeast extract, $20 \mathrm{~g} \mathrm{~L}^{-1}$ peptone, and $20 \mathrm{~g} \mathrm{~L}^{-1}$ glucose The recipe of Delft medium was described previously ${ }^{87} .100 \mathrm{mg} \mathrm{L}^{-1}$ histidine and/or $100 \mathrm{mg} \mathrm{L}^{-1}$ uracil were supplemented when needed. The SD-Ura medium contains $6.7 \mathrm{~g} \mathrm{~L}^{-1}$ yeast nitrogen base without amino acids (YNB, Formedium), $770 \mathrm{mg} \mathrm{L}^{-1}$ complete supplement mixture without uracil (CSM-URA, Formedium) and $20 \mathrm{~g} \mathrm{~L}^{-1}$ glucose. For SD-Leu medium, $770 \mathrm{mg} \mathrm{L}^{-1} \mathrm{CSM}$-URA in SD-Ura medium was replaced by $690 \mathrm{mg} \mathrm{L}^{-1}$ complete supplement mixture without leucine (CSM-LEU, Formedium). Cells with the MET25 controlled expression cassette for a-synucleinEGFP were cultured in Delft medium with $1 \mathrm{mM}$ methionine. The $\alpha$-synuclein-EGFP expression was induced by depletion of methionine from the medium. Yeast cells were cultivated at $22^{\circ} \mathrm{C}$ for essential temperature sensitive alleles or $30^{\circ} \mathrm{C}$ if not specified. Cell density $\left(\mathrm{OD}_{600 \mathrm{~nm}}\right)$ was measured using a GENESYS20 spectrophotometry (Thermo Fisher Scientific). 
SGA analysis. The S.cerevisiae strain Y7092 was used as the SGA starting strain. Query strains were constructed by chromosomally integrating two copies of GPDp$\mathrm{A} \beta 42$-CYC1t fragment (sample) with the antibiotic resistance genes (clonNat and hygromycin B) into Y7092 genome. The yeast single gene knock-out collection (SGA-V2) and the essential gene temperature sensitive allele collection (ts-V6) were kindly provided by Prof. Charlie Boone, University of Toronto, Canada. Thereafter, SGA mating was performed in biological duplicate as previously described ${ }^{28}$ to introduce the $\mathrm{A} \beta 42$ cassettes into the SGA-V2 and ts-V6 collections. A control set of the same collections was used as a control for the toxicity assay, which were constructed by introducing two copies of the GPDp-CYClt fragment with the same antibiotic resistance genes. All the pinning steps for collection handling were performed using a SINGER ROTOR HDA Robot (Singer Instrument). To define genetic interactions, colony sizes of mutants with $A \beta 42$ expression were quantified from images of final selection plates, and fitness scores relative to mutants with control were calculated via online SGAtools (http://sgatools.ccbr. utoronto.ca// ${ }^{88}$. A score of $>0$ represented positive interaction, i.e., increased colony size. While a score of $<0$ represented negative interaction, i.e., decreased colony size. Based on these scores, mutants with significantly $(p$-adj $<0.05)$ affected A $\beta 42$ toxicity were analyzed for global genetic interactions via spatial analysis of functional enrichment (SAFE) package ${ }^{30}$. Adjusted $P<0.05$ and scores $\leq-0.2$ or $\geq 0.2$ were set as thresholds to identify significantly affected mutants. Enrichment of GO terms in gene sets of interests was analyzed using the GOstats package ${ }^{89}$.

Verification of the putative interactions. To confirm the phenotype observed in SGA screening, mutants that showed significant differences between $\mathrm{A} \beta 42$ and control were tested manually by random spore analyses and complementation assays. For Random spore analysis ${ }^{28}$, spores were first resuspended in $1 \mathrm{ml}$ of sterile $\mathrm{H}_{2} \mathrm{O}$, then $40,80,80$, and $160 \mu \mathrm{l}$ of resuspended cells were plated onto agar plates selecting for the haploid, the single gene mutation, the query-gene expression (A 342 or control), and both single gene mutation and query-gene expression, respectively. Plates were incubated at $30^{\circ} \mathrm{C}$ for $2-3$ days. Cell growth under the four cultural conditions were compared between $\mathrm{A} \beta 42$ and control. Mutants with the $A \beta 42$ expression of which few or no haploids grew on plates were scored as synthetically lethal. Mutants with the A $\beta 42$ expression of which less haploids grew on plates comparing to mutants with control were scored as synthetically sick. Mutants with the $A \beta 42$ expression of which more haploids grew on plates compared to mutants with control were scored as synthetically positive. Complementation assays were performed with a selected group of mutants with $A \beta 42 /$ control expression which were complemented with the corresponding MoBY plasmids or the empty control vector (p5586). Serial dilutions (10-fold) of each transformant were grown on SD-Ura agar plates at $30^{\circ} \mathrm{C}$ for $2-3$ days.

Analysis of human brain gene expression data. We analyzed brain expression datasets from control (N) and AD cases (GN314, GN326, GN327 and GN328) deposited in the GeneNetwork website (http://www.genenetwork.org) to define the target gene expression and associate them with $\mathrm{AD}$. The study enlisted approximately $400 \mathrm{AD}$ cases and 170 controls matched for age, gender and post mortem interval. Three brain regions (cerebellum, visual cortex, and dorsolateral prefrontal cortex) from the same individuals were profiled on a custom-made Agilent $44 \mathrm{~K}$ microarray. The individuals were genotyped on two different platforms, the Illumina HumanHap650Y array and a custom Perlegen $300 \mathrm{~K}$ array. Differential expressed genes between control and AD cases were then identified using a MannWhitney test.

NADP(H) and NAD(H) measurements. Enzymatic cycling assays to determine $\mathrm{NADP}(\mathrm{H})$ and $\mathrm{NAD}(\mathrm{H})$ levels were performed according to the $\mathrm{NADP}^{+} / \mathrm{NADPH}$ Quantification Kit (Sigma) and NAD ${ }^{+} / \mathrm{NADH}$ Quantification Kit (Sigma). Cells were grown in Delft $+100 \mathrm{mg} \mathrm{L}^{-1}$ histidine $+100 \mathrm{mg} \mathrm{L}^{-1}$ uracil medium, then 5 $\mathrm{OD}_{600 \mathrm{~nm}}$ of cells were collected at later exponential $(\mathrm{EX})$ phase $\left(\mathrm{OD}_{600 \mathrm{~nm}} \approx 2\right)$ and post-diauxic (PD) phases. Collected cells were immediately added to $20 \mathrm{ml}$ of cold methanol (pre-chilled to $-80^{\circ} \mathrm{C}$ ) to quench cellular metabolism. Cells were centrifuged at $-10^{\circ} \mathrm{C}$ for $4 \mathrm{~min}(4000 \times g)$, then supernatant was removed. Cell pellets were freeze-dried for $2 \mathrm{~h}$ and stored at $-80^{\circ} \mathrm{C}$ until measurement. The dried cell pellet was resuspended in $500 \mu \mathrm{l}$ of extraction buffer (Sigma) and added to $0.2 \mathrm{~g}$ of prechilled glass beads (425-600 $\mu \mathrm{m}$ diameter, Sigma). Cells were lysed via four rounds of vortexing for $20 \mathrm{~s}$ at speed $6200 \mathrm{rpm}$ on the Precellys Evolution Homogenizer (Bertin technologies) followed by incubation for $1 \mathrm{~min}$ on ice. The cell lysates were centrifuged at $14000 \times g$ for $1 \mathrm{~min}$ at $0{ }^{\circ} \mathrm{C}$, and supernatant was used to measure $\mathrm{NADH}(\mathrm{H})$ and $\mathrm{NAD}(\mathrm{H})$ amounts following the manufacturer's instructions (Sigma).

$\mathbf{H}_{\mathbf{2}} \mathbf{O}_{\mathbf{2}}$ treatment. For growth assays, the $\mathrm{A} \beta 42$ strain was grown to mid exponential phase $\left(\mathrm{OD}_{600 \mathrm{~nm}} \approx 0.5-0.6\right)$ in Delft $+100 \mathrm{mg} \mathrm{L}^{-1}$ histidine $+100 \mathrm{mg} \mathrm{L}^{-1}$ uracil medium, the HTT103QP strain was grown to early exponential phase $\left(\mathrm{OD}_{600 \mathrm{~nm}} \approx\right.$ $0.2)$ in Delft $+100 \mathrm{mg} \mathrm{L}^{-1}$ histidine medium, and the $\alpha$-syn strain was grown to early exponential phase $\left(\mathrm{OD}_{600 \mathrm{~nm}} \approx 0.2\right)$ in Delft $+100 \mathrm{mg} \mathrm{L}^{-1}$ uracil medium, then $1 \mathrm{ml}$ of cells were distributed on 48-well MTP flowerPlates (m2p-labs). Different concentrations of $\mathrm{H}_{2} \mathrm{O}_{2}$ were then added to the cells. Cell growth was monitored for biological duplicates using a BioLector reader (m2p-labs) and analyzed with $\mathrm{R} /$ growthrates package.

For $\mathrm{H}_{2} \mathrm{O}_{2}$ spotting tests, the control strain was grown to stationary phase. 1 $\mathrm{OD}_{600 \mathrm{~nm}}$ of cells were pelleted and spotted at a series of 5-fold dilutions onto the Delft $+100 \mathrm{mg} \mathrm{L}^{-1}$ histidine $+100 \mathrm{mg} \mathrm{L}^{-1}$ uracil agar plates containing different concentrations of $\mathrm{H}_{2} \mathrm{O}_{2}$. Plates were then imaged after incubation at $30^{\circ} \mathrm{C}$ for 2-3 days.

Quantitative real-Time PCR (qPCR) analysis. $\mathrm{QPCR}$ was performed as previously described $^{90}$. A $\beta 42$ and control strains were grown in Delft medium supplemented with $100 \mathrm{mg} \mathrm{L}^{-1}$ histidine and $100 \mathrm{mg} \mathrm{L}^{-1}$ uracil. Samples were taken from biological triplicate cultures during exponential phase and after 1,2,3 days of cultivation. ACT1 was used as a reference gene to normalize RNA levels with primers ACT-L/ ACT-R. FMN1 gene expression was measured with primers FMN1-L2/FMN1-R2. Fold changes were calculated according to FMN1 gene expression in control strain and $\mathrm{A} \beta 42$ strain, respectively, during exponential phase.

FMN and FAD supplementation. Riboflavin $5^{\prime}$-monophosphate sodium salt (FMN, Sigma) or riboflavin adenine dinucleotide disodium salt (FAD, Sigma) was prepared in distilled $\mathrm{H}_{2} \mathrm{O}$ to $70 \mathrm{mM}$ stock solutions, which was filter-sterilized using $0.2 \mu \mathrm{m}$ cellulose acetate filters (VWR). Yeast strains were grown overnight, diluted to an initial $\mathrm{OD}_{600 \mathrm{~mm}}$ of 0.1 in Delft medium or SD-Ura medium. FMN stock solution was added to the culture to different final concentrations for continuous treatment. For agar plates, FMN and FAD stock solutions were added to the final concentration of $5 \mathrm{mM}$, respectively.

Viability measurement. Viability was measured by propidium iodine (PI, Thermo Fisher Scientific) staining as described previously $23.0 .5 \mathrm{OD}_{600 \mathrm{~nm}}$ of cells were taken at different culture ages (days) and stained with $0.5 \mu \mathrm{g} \mathrm{ml}^{-1}$ of PI in the dark at room temperature for $20 \mathrm{~min}$. The cell pellet was washed twice with PBS and resuspended in $1 \mathrm{ml}$ of PBS. Fluorescence was analyzed using a Guava flow cytometer (Merck). In all, 5000 cells were analyzed for each sample. Cells were also imaged under a fluorescence microscope (Leica DMI4000B) with DIC and RFP filters. Experiments were performed in biological triplicates.

Inclusion body morphology test. WT Hsp104-GFP and fmn1 Hsp104-GFP strains were grown to mid exponential phase $\left(\mathrm{OD}_{600 \mathrm{~nm}} \approx 0.5\right)$ in SD-Leu medium with G418 supplement and subjected to a heat shock at $38^{\circ} \mathrm{C}$ for $90 \mathrm{~min}$ to induce protein aggregation. Samples were taken after $90 \mathrm{~min}$ of heat shock and fixed immediately in $3.7 \%$ formaldehyde. Cells were then imaged with a Carl Zeiss axiovert $200 \mathrm{M}$ microscope (Zeiss). Images were processed with ImageJ (NIH), and at least 500 cells with aggregates were analyzed and quantified for each sample. Data is based on the average of six biological replicates.

AZE treatment. L-azetidine-2-carboxylic acid (AZE), an analogue of proline, is incorporated competitively with proline into proteins during protein synthesis, and eventually causing conformational changes and protein misfolding ${ }^{91}$. The control strain was grown to mid exponential phase $\left(\mathrm{OD}_{600 \mathrm{~nm}} \approx 0.5-0.6\right)$ in Delft medium supplemented with $100 \mathrm{mg} \mathrm{L}^{-1}$ histidine and $100 \mathrm{mg} \mathrm{L}^{-1}$ uracil. AZE (Sigma) was added to the medium to final concentrations of $5 \mathrm{mM}$ or $10 \mathrm{mM}$. After $3 \mathrm{~h}$ of cultivation at $30{ }^{\circ} \mathrm{C}, 0.2 \mathrm{OD}_{600 \mathrm{~nm}}$ of cells were pelleted and resuspended in $1 \mathrm{ml}$ of sterile $\mathrm{H}_{2} \mathrm{O}$. Serial dilutions $\left(10^{-1}, 10^{-2}, 10^{-3}\right)$ were used for spot tests on Delft + $100 \mathrm{mg} \mathrm{L}^{-1}$ histidine $+100 \mathrm{mg} \mathrm{L}^{-1}$ uracil agar plates. Viability was also determined by colony forming units (CFU) counting. In all, 400 cells were plated on Delft + histidine + uracil agar plates. Plates were incubated at $30^{\circ} \mathrm{C}$ for $2-3$ days. Results are from biological triplicates.

Western blot. $A \beta 42$ and control strains were grown to stationary phase in Delft medium supplemented with $100 \mathrm{mg} \mathrm{L}^{-1}$ histidine and $100 \mathrm{mg} \mathrm{L}^{-1}$ uracil. In all, 5 $\mathrm{OD}_{600 \mathrm{~nm}}$ of cells were collected. Whole cell protein extraction and western blotting were carried out following previous protocols ${ }^{21}$. A $\beta 42$ levels were identified by a mouse monoclonal anti-A $\beta$ antibody (6E10, Covance, Catalog Number: SIG-39320, 1:2000 dilution). Anti-GAPDH (Santa Cruz, Catalog Number: sc-365062, 1:2000 dilution) was included as a loading control.

Transcriptome profiling. Samples for RNA-seq analysis were taken from biological triplicates of cells grown until late EX phase $\left(\mathrm{OD}_{600 \mathrm{~nm}} \approx 2\right)$. In all, $10 \mathrm{OD}_{600 \mathrm{~nm}}$ of cells were collected in $50 \mathrm{ml}$ falcon tubes containing $25 \mathrm{ml}$ ice and snap-frozen in liquid nitrogen to prevent mRNA turnover and RNA extraction was performed as described previously ${ }^{23}$. RNA-seq was performed by the Novo Nordisk Foundation Center for Biosustainability of Technical University of Denmark (DTU). The Illumina TruSeq samples preparation kit v2, with poly-A enrichment, was used for RNA sequencing. The fragments were clustered on $\mathrm{cBot}$ and sequenced on a single lane on a HiSeq 4000 with paired ends $(2 \times 150 \mathrm{bp})$, according to the manufacturer's instructions. The number of read pairs ranged from 12.0 to 16.0 million for each sample. Raw RNA-seq data was preprocessed by Nfcore-RNA-seq pipeline (https://github.com/nf-core/rnaseq), developed by the National Genomics Infrastructure at SciLifeLab, Karolinska Institute. The Report GO terms were performed 
using the Platform for Integrative Analysis of Omics (PIANO) R package ${ }^{92}$ with information from the Saccharomyces Genome Database (https://www. yeastgenome.org/). The functional enrichment of KEGG pathways was analyzed using the Database for Annotation, Visualization and Integrated Discovery (DAVID, https://david.ncifcrf.gov/ $)^{93,94}$. The differential gene expression $\left(\log _{2^{-}}\right.$ FoldChange) and corresponding significance ( $p$-adj) were calculated by the Benjamini-Hochberg method and used as input. Heatmaps of significantly changed GO terms and genes were generated by using pheatmap R package.

Statistics. Significance of differences observed between strains were determined as mean \pm SD using two-tailed student $t$-test. Three biological replicates were analyzed unless specified explicitly. Statistical significance was indicated as $p<0.05$ unless specific explicitly.

Reporting summary. Further information on research design is available in the Nature Research Reporting Summary linked to this article.

\section{Data availability}

The RNA-seq raw data can be downloaded from the Genome Expression Omnibus website (GEO, https://www.ncbi.nlm.nih.gov/geo/) with series number GSE128905. The data that support the findings of this study are available from the corresponding author upon reasonable request. The source data underlying Figs. 3c, e, 4a, b, d, 5, 7 and Supplementary Figs. 11a, 13a, 20, and 22 are provided as a Source Data file.

Received: 24 April 2019; Accepted: 7 January 2020;

Published online: 13 February 2020

\section{References}

1. Wyss-Coray, T. Ageing, neurodegeneration and brain rejuvenation. Nature 539, 180-186 (2016).

2. Selkoe, D. J. \& Hardy, J. The amyloid hypothesis of Alzheimer's disease at 25 years. EMBO Mol. Med. 8, 595-608 (2016).

3. Meyer-Luehmann, M. et al. Exogenous induction of cerebral betaamyloidogenesis is governed by agent and host. Science 313, 1781-1784 (2006).

4. Gouras, G. K., Almeida, C. G. \& Takahashi, R. H. Intraneuronal Abeta accumulation and origin of plaques in Alzheimer's disease. Neurobiol. Aging. 26, 1235-1244 (2005).

5. Graham, W. V., Bonito-Oliva, A. \& Sakmar, T. P. Update on Alzheimer's Disease therapy and prevention strategies. Annu. Rev. Med. 68, 413-430 (2017).

6. Dubnikov, T., Ben-Gedalya, T. \& Cohen, E. Protein quality control in health and disease. Cold Spring Harb. Perspect. Biol. 9, a023523 (2017).

7. Duran-Aniotz, C., Martínez, G. \& Hetz, C. Memory loss in Alzheimer's disease: are the alterations in the UPR network involved in the cognitive impairment? Front. Aging Neurosci. 6, 8 (2014).

8. Hetz, C. The unfolded protein response: controlling cell fate decisions under ER stress and beyond. Nat. Rev. Mol. Cell Biol. 13, 89-102 (2012).

9. Sitia, R. \& Braakman, I. Quality control in the endoplasmic reticulum protein factory. Nature 426, 891-894 (2003).

10. Meusser, B., Hirsch, C., Jarosch, E. \& Sommer, T. ERAD: the long road to destruction. Nat. Cell Biol. 7, 766-772 (2005).

11. Woehlbier, U. \& Hetz, C. Modulating stress responses by the UPRosome: a matter of life and death. Trends Biochem.Sci. 36, 329-337 (2011).

12. Cisse, M., Duplan, E. \& Checler, F. The transcription factor XBP1 in memory and cognition: Implications in Alzheimer disease. Mol. Med. 22, 905-917 (2017).

13. Ohno, M. Roles of eIF2a kinases in the pathogenesis of Alzheimer's disease. Front. Mol. Neurosci. 7, 22 (2014).

14. Matlack, K. E. et al. Clioquinol promotes the degradation of metal-dependent amyloid-beta (Abeta) oligomers to restore endocytosis and ameliorate Abeta toxicity. Proc. Natl Acad. Sci. USA 111, 4013-4018 (2014).

15. Hill, S. M., Hao, X., Liu, B. \& Nystrom, T. Life-span extension by a metacaspase in the yeast Saccharomyces cerevisiae. Science 344, 1389-1392 (2014).

16. Macheroux P., Kappes B., Ealick S. E. Flavogenomics-a genomic and structural view of flavin-dependent proteins. FEBS J. 278, 2625-2634 (2011).

17. Nalivaeva, N. N. \& Turner, A. J. The amyloid precursor protein: A biochemical enigma in brain development, function and disease. FEBS Lett. 587, 2046-2054 (2013).

18. Haass C., Kaether C., Thinakaran G., Sisodia S. Trafficking and proteolytic processing of APP. Cold Spring Harb. Perspect. Med. 2, a006270 (2012).

19. Treusch, S. et al. Functional links between Abeta toxicity, endocytic trafficking, and Alzheimer's disease risk factors in yeast. Science 334, 1241-1245 (2011).
20. D’Angelo, F. et al. A yeast model for amyloid-beta aggregation exemplifies the role of membrane trafficking and PICALM in cytotoxicity. Dis. Model. Mech. 6, 206-216 (2013)

21. Chen, X. \& Petranovic, D. Amyloid-beta peptide-induced cytotoxicity and mitochondrial dysfunction in yeast. FEMS Yeast Res. 15, fov061 (2015).

22. Luheshi, L. M. et al. Systematic in vivo analysis of the intrinsic determinants of Amyloid $\beta$ pathogenicity. PLOS Biol. 5, e290 (2007).

23. Chen, $X$. et al. Interplay of energetics and ER stress exacerbates Alzheimer's Amyloid-beta (Abeta) toxicity in Yeast. Front. Mol. Neurosci. 10, 232 (2017).

24. Yang, J., Hao, X., Cao, X., Liu, B. \& Nyström, T. Spatial sequestration and detoxification of Huntingtin by the ribosome quality control complex. eLife $\mathbf{5}$, e11792 (2016)

25. Zhao, L. et al. A genome-wide imaging-based screening to identify genes involved in synphilin-1 inclusion formation in Saccharomyces cerevisiae. Sci. Rep. 6, 30134 (2016).

26. Nair, S., Traini, M., Dawes, I. W. \& Perrone, G. G. Genome-wide analysis of Saccharomyces cerevisiae identifies cellular processes affecting intracellular aggregation of Alzheimer's amyloid- $\beta 42$ : importance of lipid homeostasis. Mol. Biol. Cell 25, 2235-2249 (2014).

27. Fruhmann, G. et al. The impact of ESCRT on Abeta1-42 induced membrane lesions in a Yeast Model for Alzheimer's Disease. Front. Mol. Neurosci. 11, 406 (2018).

28. Tong, A. H. \& Boone, C. Synthetic genetic array analysis in Saccharomyces cerevisiae. Methods Mol. Biol. 313, 171-192 (2006).

29. Costanzo, M. et al. A global genetic interaction network maps a wiring diagram of cellular function. Science 353, aaf1420 (2016).

30. Baryshnikova, A. Spatial analysis of functional enrichment (SAFE) in large biological networks. Methods Mol. Biol. 1819, 249-268 (2018)

31. Zhao, Y., Tan, W., Sheng, W. \& Li, X. Identification of biomarkers associated with alzheimer's disease by bioinformatics analysis. Am. J. Alzheimers Dis. Other Demen. 31, 163-168 (2016).

32. Trychta, K. A., Back, S., Henderson, M. J. \& Harvey, B. K. KDEL receptors are differentially regulated to maintain the ER proteome under calcium deficiency. Cell Rep. 25, 1829-1840.e1826 (2018).

33. Park, H. J. et al. Retention in endoplasmic reticulum 1 (RER1) modulates amyloid-beta (Abeta) production by altering trafficking of gamma-secretase and amyloid precursor protein (APP). J. Biol. Chem. 287, 40629-40640 (2012).

34. Shi, Y. et al. Rpn1 provides adjacent receptor sites for substrate binding and deubiquitination by the proteasome. Science 351, aad9421 (2016).

35. Santos, M. A., Jimenez, A. \& Revuelta, J. L. Molecular characterization of FMN1, the structural gene for the monofunctional flavokinase of Saccharomyces cerevisiae. J. Biol. Chem. 275, 28618-28624 (2000).

36. Marashly, E. T. \& Bohlega, S. A. Riboflavin has neuroprotective potential: focus on Parkinson's disease and migraine. Front. Neurol. 8, 333 (2017).

37. Santos M. A., García-Ramírez J. J., Revuelta J. L. Riboflavin Biosynthesis in Saccharomyces cerevisiae: cloning, characterization, and expression of the rib5 gene encoding riboflavin synthase. J. Biol. Chem. 270, 437-444 (1995).

38. Karthikeyan, S. et al. Crystal structure of human riboflavin kinase reveals a beta barrel fold and a novel active site arch. Structure 11, 265-273 (2003).

39. Haass, C. \& Selkoe, D. J. Soluble protein oligomers in neurodegeneration: lessons from the Alzheimer's amyloid beta-peptide. Nat. Rev. Mol. cell Biol. 8, 101-112 (2007).

40. Glover, J. R. \& Lindquist, S. Hsp104, Hsp70, and Hsp40: a novel chaperone system that rescues previously aggregated proteins. Cell 94, 73-82 (1998).

41. Hill Sandra, M. et al. Asymmetric inheritance of aggregated proteins and age reset in yeast are regulated by Vac17-dependent vacuolar functions. Cell Rep. 16, 826-838 (2016).

42. Kitamura, A. et al. Cytosolic chaperonin prevents polyglutamine toxicity with altering the aggregation state. Nat. Cell Biol. 8, 1163-1170 (2006).

43. Lobo, V., Patil, A., Phatak, A. \& Chandra, N. Free radicals, antioxidants and functional foods: Impact on human health. Pharmacogn. Rev. 4, 118-126 (2010).

44. Liguori, I. et al. Oxidative stress, aging, and diseases. Clin. Interv. Aging 13, 757-772 (2018)

45. Krüger A., et al. Tpol-mediated spermine and spermidine export controls cell cycle delay and times antioxidant protein expression during the oxidative stress response. EMBO Rep. 14, 1113-1119 (2013).

46. Barbara, J. H., Rikke, K. O., Peter, B. \& Claudio, M. G. Emerging roles for riboflavin in functional rescue of mitochondrial $\beta$-oxidation flavoenzymes. Curr. Med. Chem. 17, 3842-3854 (2010).

47. Qiao, K., Wasylenko, T. M., Zhou, K., Xu, P. \& Stephanopoulos, G. Lipid production in Yarrowia lipolytica is maximized by engineering cytosolic redox metabolism. Nat. Biotechnol. 35, 173 (2017).

48. Lewis, D. A. \& Bisson, L. F. The HXT1 gene product of Saccharomyces cerevisiae is a new member of the family of hexose transporters. Mol. Cell. Biol. 11, 3804-3813 (1991).

49. Ozcan, S. \& Johnston, M. Function and regulation of yeast hexose transporters. Microbiol. Mol. Biol. Rev. 63, 554-569 (1999). 
50. Felix, C. F. et al. Protection against thermal denaturation by trehalose on the plasma membrane $\mathrm{H}+$-ATPase from yeast. Synergetic effect between trehalose and phospholipid environment. Eur. J. Biochem. 266, 660-664 (1999).

51. Liu, R., Barkhordarian, H., Emadi, S., Park, C. B. \& Sierks, M. R. Trehalose differentially inhibits aggregation and neurotoxicity of beta-amyloid 40 and 42. Neurobiol. Dis. 20, 74-81 (2005).

52. Gudipati, V., Koch, K., Lienhart, W. D. \& Macheroux, P. The flavoproteome of the yeast Saccharomyces cerevisiae. Biochim. et. biophys. Acta 1844, 535-544 (2014).

53. Mittelstadt, $M$. et al. Interaction of human tRNA-dihydrouridine synthase-2 with interferon-induced protein kinase PKR. Nucleic acids Res. 36, 998-1008 (2008).

54. Onuki, R. et al. An RNA-dependent protein kinase is involved in tunicamycininduced apoptosis and Alzheimer's disease. EMBO J. 23, 959-968 (2004).

55. Mangiarini, L. et al. Exon 1 of the HD gene with an expanded CAG repeat is sufficient to cause a progressive neurological phenotype in transgenic mice. Cell 87, 493-506 (1996).

56. Krobitsch, S. \& Lindquist, S. Aggregation of huntingtin in yeast varies with the length of the polyglutamine expansion and the expression of chaperone proteins. Proc. Natl Acad. Sci. USA 97, 1589-1594 (2000).

57. Kumar, A. \& Ratan, R. R. Oxidative stress and Huntington's disease: the good, the bad, and the ugly. J. Huntingt. Dis. 5, 217-237 (2016).

58. Peelaerts, W. et al. a-Synuclein strains cause distinct synucleinopathies after local and systemic administration. Nature 522, 340 (2015).

59. Zabrocki P., et al. Characterization of $a$-synuclein aggregation and synergistic toxicity with protein tau in yeast. FEBS J. 272, 1386-1400 (2005).

60. Dey, S. \& Bishayi, B. Riboflavin along with antibiotics balances reactive oxygen species and inflammatory cytokines and controls Staphylococcus aureus infection by boosting murine macrophage function and regulates inflammation. J. Inflamm. 13, 36 (2016).

61. Ashoori, M. \& Saedisomeolia, A. Riboflavin (vitamin B2) and oxidative stress: a review. Br. J. Nutr. 111, 1985-1991 (2014).

62. Durusoy, M., Karagoz, E. \& Ozturk, K. Assessment of the relationship between the antimutagenic action of riboflavin and glutathione and the levels of antioxidant enzymes. J. Nutr. Biochem. 13, 598-602 (2002).

63. $\mathrm{Yu}, \mathrm{Z}$. et al. Endogenous superoxide dismutase activation by oral administration of riboflavin reduces abdominal aortic aneurysm formation in rats. J. Vasc. Surg. 64, 737-745 (2016).

64. Lin, M. T. \& Beal, M. F. Mitochondrial dysfunction and oxidative stress in neurodegenerative diseases. Nature 443, 787 (2006)

65. Scholte, H. R. et al. Riboflavin-responsive complex I deficiency. Biochim. et Biophys. Acta 1271, 75-83 (1995).

66. Bugiani, M. et al. Effects of riboflavin in children with complex II deficiency. Brain Dev. 28, 576-581 (2006).

67. Swerdlow, R. H. Mitochondria and mitochondrial cascades in Alzheimer's Disease. J. Alzheimer's Dis. 62, 1403-1416 (2018)

68. Sonntag, K.-C. et al. Late-onset Alzheimer's disease is associated with inherent changes in bioenergetics profiles. Sci. Rep. 7, 14038 (2017).

69. Yao, J. et al. Mitochondrial bioenergetic deficit precedes Alzheimer's pathology in female mouse model of Alzheimer's disease. Proc. Natl Acad. Sci. USA 106, 14670-14675 (2009).

70. Ghosh D., Levault K. R., Brewer G. J. Relative importance of redox buffers GSH and NAD(P)H in age-related neurodegeneration and Alzheimer diseaselike mouse neurons. Aging Cell. 13, 631-640 (2014).

71. Hübel, F., Steegborn, C., Sprinzl, M. \& Steinberg, S. Compilation of tRNA Sequences and Sequences of tRNA Genes. Nucleic Acids Res. 24, 68-72 (1996).

72. Lienhart, W.-D., Gudipati, V. \& Macheroux, P. The human flavoproteome. Arch. Biochem. Biophys. 535, 150-162 (2013).

73. Kato, T. et al. A novel human tRNA-Dihydrouridine synthase involved in pulmonary. Carcinogenesis 65, 5638-5646 (2005)

74. Miller, S. B. M., Mogk, A. \& Bukau, B. Spatially organized aggregation of misfolded proteins as cellular stress defense strategy. J. Mol. Biol. 427, 1564-1574 (2015).

75. Thibault, G., Ismail, N. \& Ng, D. T. The unfolded protein response supports cellular robustness as a broad-spectrum compensatory pathway. Proc. Natl Acad. Sci. USA 108, 20597-20602 (2011).

76. Hwang, C., Sinskey, A. J. \& Lodish, H. F. Oxidized redox state of glutathione in the endoplasmic reticulum. Science 257, 1496-1502 (1992).

77. Appenzeller-Herzog, C. Glutathione- and non-glutathione-based oxidant control in the endoplasmic reticulum. J. Cell Sci. 124, 847-855 (2011).

78. Willingham, S., Outeiro, T. F., DeVit, M. J., Lindquist, S. L. \& Muchowski, P. J. Yeast genes that enhance the toxicity of a mutant huntingtin fragment or $a-$ synuclein. Science 302, 1769-1772 (2003).

79. Zheng, J. et al. Role of the ribosomal quality control machinery in nucleocytoplasmic translocation of polyQ-expanded huntingtin exon-1. Biochem. Biophys. Res. Commun. 493, 708-717 (2017).

80. Andersen, J. Oxidative stress in neurodegeneration: cause or consequence? Nat. Med. 10, S18-S25 (2004).
81. Rojas-Gutierrez E., et al. Alzheimer's disease and metabolic syndrome: a link from oxidative stress and inflammation to neurodegeneration. Synapse. 71, e21990 (2017).

82. Ho, C. H. et al. A molecular barcoded yeast ORF library enables mode-ofaction analysis of bioactive compounds. Nat. Biotechnol. 27, 369-377 (2009).

83. Zhu, Z. et al. Enabling the synthesis of medium chain alkanes and 1-alkenes in yeast. Metab. Eng. 44, 81-88 (2017).

84. Naito, Y., Hino, K., Bono, H. \& Ui-Tei, K. CRISPRdirect: software for designing CRISPR/Cas guide RNA with reduced off-target sites. Bioinformatics 31, 1120-1123 (2015).

85. Berglund, L. L., Hao, X., Liu, B., Grantham, J. \& Nyström, T. Differential effects of soluble and aggregating polyQ proteins on cytotoxicity and type-1 myosin-dependent endocytosis in yeast. Sci. Rep. 7, 11328 (2017).

86. Gietz, R. D. \& Schiestl, R. H. High-efficiency yeast transformation using the LiAc/SS carrier DNA/PEG method. Nat. Protoc. 2, 31-34 (2007).

87. Maury, J. et al. EasyClone: method for iterative chromosomal integration of multiple genes Saccharomyces cerevisiae. FEMS Yeast Res. 14, 238-248 (2014).

88. Wagih, O. et al. SGAtools: one-stop analysis and visualization of array-based genetic interaction screens. Nucleic Acids Res. 41, W591-W596 (2013).

89. Falcon, S. \& Gentleman, R. Using GOstats to test gene lists for GO term association. Bioinformatics 23, 257-258 (2006).

90. Munoz-Arellano, A. J., Chen, X., Molt, A., Meza, E. \& Petranovic, D. Different expression levels of human mutant ubiquitin $\mathrm{B}(+1)(\mathrm{UBB}(+1))$ can modify chronological lifespan or stress resistance of Saccharomyces cerevisiae. Front. Mol. Neurosci. 11, 200 (2018).

91. Zagari, A., Némethy, G. \& Scheraga, H. A. The effect of the L-azetidine-2 carboxylic acid residue on protein conformation. III. Collagen-like poly (tripeptide)s. Biopolymers 30, 951-959 (1990).

92. Varemo, L., Nielsen, J. \& Nookaew, I. Enriching the gene set analysis of genome-wide data by incorporating directionality of gene expression and combining statistical hypotheses and methods. Nucleic Acids Res. 41, 4378-4391 (2013).

93. Huang da, W., Sherman, B. T. \& Lempicki, R. A. Systematic and integrative analysis of large gene lists using DAVID bioinformatics resources. Nat. Protoc. 4, 44-57 (2009).

94. Huang da, W., Sherman, B. T. \& Lempicki, R. A. Bioinformatics enrichment tools: paths toward the comprehensive functional analysis of large gene lists. Nucleic Acids Res. 37, 1-13 (2009).

\section{Acknowledgements}

D.P. would like to dedicate the paper to the memory of Prof. Susan Lindquist, a dear friend, mentor, and colleague whose work on humanized yeast models and proteostasis continues to inspire us. The work was supported by the Novo Nordisk Foundation Center for Biosustainability (21210022) and VINNOVA center CellNova (2017-02105). We would like to thank Professor Charles Boone (University of Toronto) for providing SGA strain collections, Dr. Beidong Liu (Gothenburg University) for providing WT Hsp104-GFP strain, Department of Chemistry \& Molecular Biology in Gothenburg University for providing MoBY plasmids, Dr. Lisa L. Berglund (Gothenburg University) for providing pYES2 HTT103QP plasmid, Professor Joris Winderickx (KU Leuven) for providing pUG23 $\alpha$-syn plasmid. We thank Dr. Kate Campbell for the help on $\mathrm{H}_{2} \mathrm{O}_{2}$ experiments and data analysis with R/growth rates package, Dr. Markus M.M. Bisschops, Dr. Kate Campbell, Dr. Zhiwe Zhu, and Dr. Quanli Liu for the valuable comments on the paper. We acknowledge the Novo Nordisk Foundation Center for Biosustainability for help with the RNA-seq experiment. Open access funding provided by Chalmers University of Technology.

\section{Author contributions}

X.C., F.E., T.N., and D.P. conceived and designed the study. X.C., X.H., and X.L. performed experiments. X.C., B.J., and X.L. analyzed the data. X.C., and D.P. wrote the paper. All authors were involved in interpretation, editing, and discussion.

\section{Competing interests}

The authors declare no competing interests.

\section{Additional information}

Supplementary information is available for this paper at https://doi.org/10.1038/s41467 020-14525-4.

Correspondence and requests for materials should be addressed to D.P.

Peer review information Nature Communications thanks Ian Macreadie and other, anonymous, reviewer(s) for their contribution to the peer review of this work.

Reprints and permission information is available at http://www.nature.com/reprints

Publisher's note Springer Nature remains neutral with regard to jurisdictional claims in published maps and institutional affiliations. 
(c) (i) Open Access This article is licensed under a Creative Commons Attribution 4.0 International License, which permits use, sharing, adaptation, distribution and reproduction in any medium or format, as long as you give appropriate credit to the original author(s) and the source, provide a link to the Creative Commons license, and indicate if changes were made. The images or other third party material in this article are included in the article's Creative Commons license, unless indicated otherwise in a credit line to the material. If material is not included in the article's Creative Commons license and your intended use is not permitted by statutory regulation or exceeds the permitted use, you will need to obtain permission directly from the copyright holder. To view a copy of this license, visit http://creativecommons.org/ licenses/by/4.0/.

(C) The Author(s) 2020 